\title{
Analytical study of the powered Swing-By maneuver for elliptical systems and analysis of its efficiency
}

\author{
Alessandra F.S. Ferreira ${ }^{1,2} \cdot$ Antônio F.B.A. Prado ${ }^{1} \cdot$ Othon C. Winter $^{2}$ (D) Denilson P.S. Santos $^{3}$ (iD
}

Received: 5 April 2018 / Accepted: 31 May 2018 / Published online: 11 June 2018

(c) Springer Nature B.V. 2018

\begin{abstract}
Analytical equations describing the velocity and energy variation of a spacecraft in a Powered Swing-By maneuver in an elliptic system are presented. The spacecraft motion is limited to the orbital plane of the primaries. In addition to gravity, the spacecraft suffers the effect of an impulsive maneuver applied when it passes by the periapsis of its orbit around the secondary body of the system. This impulsive maneuver is defined by its magnitude $\delta V$ and the angle that defines the direction of the impulse with respect to the velocity of the spacecraft $(\alpha)$. The maneuver occurs in a system of main bodies that are in elliptical orbits, where the velocity of the secondary body varies according to its position in the orbit following the rules of an elliptical orbit. The equations are dependent on this velocity. The study is done using the "patched-conics approximation", which is a method of simplifying the calculations of the trajectory of a spacecraft traveling around more than one celestial body. Solutions for the velocity and energy variations as a function of the parameters that define the maneuver are presented. An analysis of the efficiency of the powered Swing-By maneu-
\end{abstract}

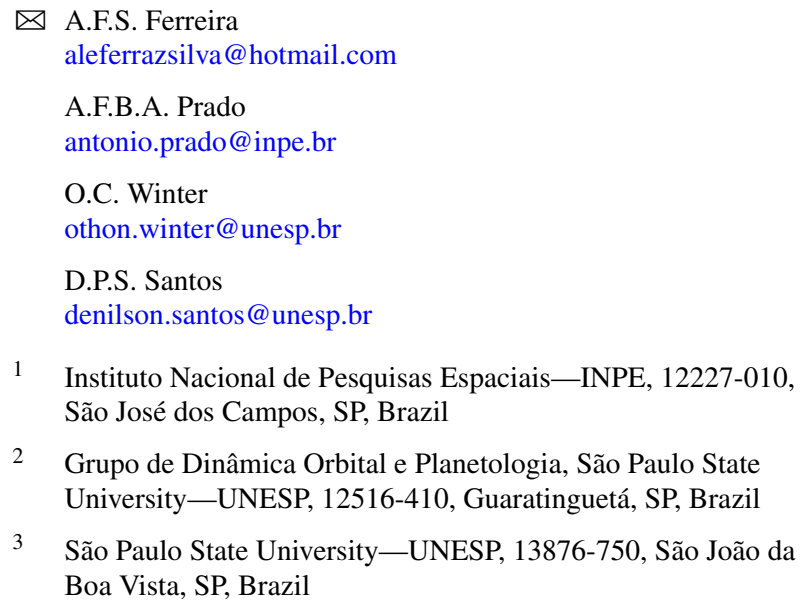

2 Grupo de Dinâmica Orbital e Planetologia, São Paulo State University-UNESP, 12516-410, Guaratinguetá, SP, Brazil

3 São Paulo State University-UNESP, 13876-750, São João da Boa Vista, SP, Brazil

ver is also made, comparing it with the pure gravity Swingby maneuver with the addition of an impulse applied outside the sphere of influence of the secondary body. After a general study, the techniques developed here are applied to the systems Sun-Mercury and Sun-Mars, which are real and important systems with large eccentricity. This problem is highly nonlinear and the dynamics very complex, but very reach in applications.

Keywords Astrodynamics · Powered Swing-By maneuvers - Spacecraft trajectories - Elliptical systems · Impulsive maneuver

\section{Introduction}

Swing-by maneuvers are used in space missions to send probes to targets like planets, moons or asteroids. The main goal of this maneuver is the fuel economy in spacecraft maneuvers. The spacecraft is sent towards the secondary body of the system and makes a close encounter with this body, taking advantage of its gravity to modify the trajectory and to move away from the body. In the case of the powered Swing-By maneuver, besides the gravity, the spacecraft suffers the effect of an impulsive maneuver applied at the moment of the closest encounter, with the objective of optimizing the maneuver.

Broucke (1988) presented an analytical study of the pure gravity Swing-By, showing the increment of velocity, energy and angular momentum, for a system of main bodies in circular orbits, and considering the dynamics given by the "patched-conics" approach. A study considering a more accurate dynamics (restricted three-body problem) is available in Prado (2007), where both models are compared for maneuvers using the planet Jupiter for the close approach. 
Later, Prado (1996) expanded this study by analyzing the addition of the application of an impulse in the spacecraft, with variable magnitude and direction, at the instant of the closest encounter. Ferreira et al. (2015) made a more complete analysis of this problem using numerical integrations. Making a more general study, Casalino et al. (1999a) presented an analytical study for Swing-By maneuvers with an impulse applied at different positions of the orbit, not only in the periapsis. The variation of the position of the application point of the impulse was made by varying the radius vector of the spacecraft with respect to the secondary body. A numerical map of the solutions for the Earth-Moon system considering the impulse applied in different directions and positions of the orbit is presented in Ferreira et al. (2017b). In this work the position of the application of the impulse is defined by the true anomaly of the spacecraft with respect to the secondary body. There are also several studies in the literature that combine low thrust, gravity assist and three body problem (Casalino et al. 1999b; McConaghy et al. 2003; Okutsu et al. 2006; Santos et al. 2008; Pourtakdoust and Sayanjali 2014; Zotos 2015; Qian et al. 2016). Regarding practical applications, Flandro (1966) projected the Voyager missions, which were sent to explore the outer planets of the solar system. The Swing-By maneuver was also applied to the missions Galileo (D'Amario et al. 1981, 1982; Byrnes and D'Amario 1982), Mariner 10 (Dunne and Burgess 1978), Messenger (McNutt et al. 2004, 2006; Grard and Mercury 2006) and LCROSS, which made a close approach to the Moon (LCROSS 2009). Studies considering gravity assist in clouds of particles are also available in the literature (Gomes and Prado 2008).

Regarding the eccentricity of the main bodies, systems of this type are numerous in the real world and there are several studies reported using this dynamics. Even some planets of the Solar System have large eccentricities. Mars is in an elliptic orbit with eccentricity 0.0934 and Mercury has an eccentricity of 0.2056 . Both planets will be used in the numerical simulations in the end of the present paper. An example of an even higher eccentric system in the Solar System is the dwarf planet Haumea, which has a moon with an eccentricity close to 0.25 (Sanchez et al. 2016). A first study of maneuvers in eccentric systems is shown in Prado (1997), which studied the pure gravity Swing-By maneuver (without impulses) for the elliptic case and Ferreira et al. (2017c, 2017a), which made a numerical study of the powered Swing-By maneuver, with the impulse applied at the periapsis of the orbit of the spacecraft around the secondary body of the system and in different directions, considering the two main bodies in elliptical orbits. An analysis of the effect of the eccentricity was presented in these papers, besides the study of the maximum gains and losses of energy obtained from the maneuver. Another possible application of maneuvers involving a close encounter and the application of impulses in highly elliptical systems occur when considering asteroids in the Solar System. These close encounters have the function of observing these bodies, so it is a scientific necessity of the mission. It means that gains and losses of energy are generated and they need to be taken into account by mission designers.

Therefore, the objective of the present paper is to derive analytical equations for the velocity and energy variations given by the powered Swing-By maneuver as a function of the basic parameters that describes the Swing-By and the parameters related to the impulsive maneuver for systems with two main bodies in elliptical orbits. The spacecraft moves in the orbital plane of the primaries. In addition, it will be presented an analysis of the efficiency of the powered Swing-By with the impulse applied at the periapsis of the orbit of the spacecraft around the secondary body of the system, compared to the pure gravity Swing-By followed by the application of the impulse after the close approach in a point far from the secondary body. This analysis shows the real limitations and gains of the powered Swing-By maneuvers. Powered Swing-By maneuvers are not used very frequent, in particular due to the risks involved if errors are made in the magnitude and/or direction of the thrust, but a general study like the one performed here can map the regions where it is advantageous, opening the way for more detailed studies for particular missions that may take advantage of this maneuver. In that sense, the goal of the present paper is to provide the literature with a general study of this maneuver, showing its advantages over more traditional maneuvers, as well as the best conditions to apply this maneuver. Real applications depend on the particularity of each mission.

\section{Statement of the problem}

The powered Swing-By maneuver is the combination of the passage of a spacecraft near the secondary body of a double system with the application of an impulsive maneuver in the spacecraft when it is passing inside the sphere of influence (Bate et al. 1971; Araujo et al. 2008) of the secondary body of the system. The impulsive maneuver is applied in the orbital plane of the motion of the two main bodies, with variable magnitude and direction. The first part of the trajectory of the spacecraft, before the application of the impulse, is similar to the equivalent part of the pure gravity SwingBy maneuver. The application of the impulse modifies the velocity and energy of the spacecraft, generating a new trajectory.

The two main bodies of the system are considered to be in elliptical orbits around their common center of mass. It is known that, in this situation, the velocity of the secondary body with respect to the primary one is dependent on the 


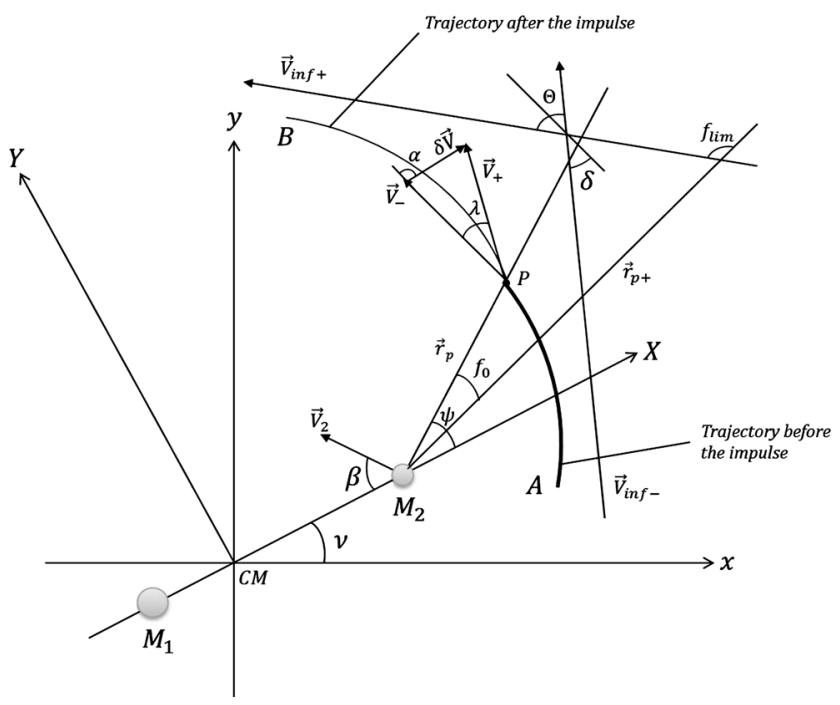

Fig. 1 Geometry of the powered Swing-By maneuver for the elliptical case (adapted from Prado 1996)

eccentricity of the orbit and the position of the secondary body in its orbit, expressed by its true anomaly.

The study is done using a method to simplify the calculations of the trajectories of the spacecraft called "patchedconics approximation". Under this assumption, each body has its own sphere of influence (Bate et al. 1971; Araujo et al. 2008). A detailed study of the errors introduced by this assumption is shown in Negri et al. (2017). When the spacecraft is within the sphere of influence of one of the bodies, only the gravitational force between the spacecraft and this body is considered, while the gravity field of the other body is neglected. This method reduces the problem of many bodies to several two-body problems. Figure 1 shows the geometry of the powered Swing-By maneuver, for the elliptical case.

$M_{1}$ and $M_{2}$ are the primary and secondary bodies of the system, respectively. These bodies are orbiting a common center of mass denoted by $C M$. The true anomaly of $M_{2}$ is $v$ and the velocity of $M_{2}$ with respect to $M_{1}$ is $\vec{V}_{2}$. The angle between $\vec{V}_{2}$ and the line connecting the $M_{1}-M_{2}$ is $180^{\circ}-\beta$. $A$ and $B$ are points of the trajectory before and after the application of the impulse, respectively, where the effect of $M_{2}$ in the spacecraft is disregarded. $P$ is the periapsis of the orbit. The magnitude of the periapsis radius of the orbit of the spacecraft is $r_{p}, \vec{V}_{-}$is its velocity at this point before the impulse and $\vec{V}_{+}$is the velocity at this point after the impulse; $r_{p+}$ is the magnitude of the periapsis radius of the spacecraft of the trajectory after the impulse and $\psi$ is the angle of approach that defines the orientation of the Swing-By; $\lambda$ is the angle between $\vec{V}_{-}$and $\vec{V}_{+}$. The vector representing the impulsive maneuver is $\delta \vec{V}$, being $\delta V$ the magnitude of the impulse and $\alpha$ the angle that defines the direction of the impulse with respect to the velocity of the spacecraft. $\vec{V}_{i n f-}$ and $\vec{V}_{i n f+}$ are the velocities of approach and departure of

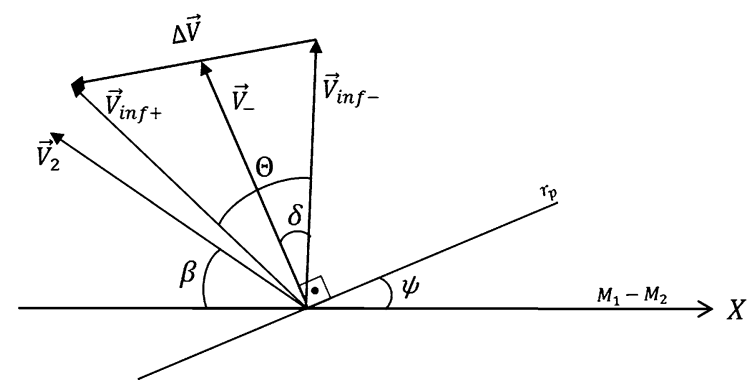

Fig. 2 Velocity vectors involved in the powered Swing-By maneuver for the elliptical case

the spacecraft relative to $M_{2}$, respectively. $\delta$ is the deflection angle of the maneuver before the impulse and $\Theta$ is the total deflection angle of the maneuver.

The spacecraft comes from an orbit around $M_{1}$, passes by the point $A$ (the velocity of the spacecraft, with respect to $M_{1}$, at this point is $\vec{V}_{i}$ ) and then it enters the sphere of influence of $M_{2}$. After that it approaches the body and receives an impulse at the moment that it is in the periapsis of its orbit around $M_{2}$ (point $P$ ). At this moment its trajectory changes immediately due to the combination of the gravity force and the impulse. The spacecraft then moves until it leaves its sphere of influence, passes by the point $B$ (the velocity at this point is $\vec{V}_{o}$ ) and leaves $M_{2}$. The whole maneuver is performed in the orbital plane of the primaries.

\subsection{Analytical equations}

First, it is necessary to obtain the variation of the velocity and energy of the spacecraft in the maneuver. Figure 2 shows a representation of the velocity vectors involved in the maneuver.

It is known that the velocity variation vector $\Delta \vec{V}$ is given by the difference between $\vec{V}_{o}$ and $\vec{V}_{i}$, where $\vec{V}_{i}=$ $\vec{V}_{i n f-}+\vec{V}_{2}$ and $\vec{V}_{o}=\vec{V}_{i n f+}+\vec{V}_{2}$. Thus, $\Delta V=$ $\sqrt{\left(V_{O X}-V_{i X}\right)^{2}+\left(V_{O Y}-V_{i Y}\right)^{2}}$. Decomposing $\vec{V}_{2}$ in the plane of motion, we get $\vec{V}_{2}=\left(-V_{2} \cos \beta, V_{2} \sin \beta\right)$. The components of the velocities $\vec{V}_{i n f-}$ and $\vec{V}_{i n f+}$ are obtained from Fig. 2.

$$
\vec{V}_{i n f-}:\left\{\begin{array}{l}
\dot{X}=V_{i n f-} \cos \left(90^{\circ}+\psi-\delta\right)=-V_{i n f-} \sin (\psi-\delta) \\
\dot{Y}=V_{i n f-} \sin \left(90^{\circ}+\psi-\delta\right)=+V_{i n f-} \cos (\psi-\delta)
\end{array}\right.
$$

$\vec{V}_{i n f+}:\left\{\begin{aligned} \dot{X}_{+} & =V_{i n f+} \cos \left(90^{\circ}-\delta+\psi+\Theta\right) \\ & =-V_{i n f+} \sin (\psi-\delta+\Theta) \\ \dot{Y}_{+} & =V_{i n f+} \sin \left(90^{\circ}-\delta+\psi+\Theta\right) \\ & =+V_{i n f+} \cos (\psi-\delta+\Theta)\end{aligned}\right.$ 
Therefore the $\vec{V}_{i}$ and $\vec{V}_{o}$ components in the plane are as follows:

$V_{i X}=-V_{i n f-} \sin (\psi-\delta)-V_{2} \cos \beta$,

$V_{i Y}=V_{\text {inf- }} \cos (\psi-\delta)+V_{2} \sin \beta$,

$V_{o X}=-V_{i n f+} \sin (\psi-\delta+\Theta)-V_{2} \cos \beta$,

$V_{o Y}=V_{i n f+} \cos (\psi-\delta+\Theta)+V_{2} \sin \beta$.

From the energy conservation principle, it is possible to calculate the magnitude of the velocity of the spacecraft in its periapsis before applying the impulse. It is $V_{-}=$ $\sqrt{V_{\text {inf- }}^{2}+\frac{2 \mu}{r_{p}}}$, where $\mu$ is the gravitational parameter of the secondary body.

The velocity of the spacecraft at the periapsis of its orbit after the impulse is given by $V_{+}=$ $\sqrt{V_{-}^{2}+\delta V^{2}+2 V_{-} \delta V \cos \alpha}$. Therefore, the magnitude of the velocity of departure of the spacecraft after the impulse is $V_{i n f+}=\sqrt{V_{+}^{2}-\frac{2 \mu}{r_{p}}}$. For the situations where $V_{+}^{2}-\frac{2 \mu}{r_{p}}<0$, the spacecraft do not escape from its orbit around the secondary body and we have a capture.

The semi-major axis of the orbit of the spacecraft around $M_{1}$ after the Swing-By is given by $a_{s}=\sqrt{\frac{\mu}{V_{i n f+}^{2}}}$, for values of $V_{i n f}+$ greater than zero. The angle between $V_{-}$and
$V_{+}$is $\lambda=\cos ^{-1}\left(\frac{\delta V^{2}-V_{-}^{2}-V_{+}^{2}}{-2 V_{-} V_{+}}\right)$. The angular momentum $h$, the semilatus rectum $p$ and the eccentricity of the second orbit can also be calculated. The equations are $h=$ $r_{p} V_{+} \sin \left(90^{\circ}-\lambda\right), p=\frac{h^{2}}{\mu}$ and $e_{s}=\sqrt{1+\frac{p}{a_{s}}}$, respectively.

Then, the true anomaly of the spacecraft around the secondary body is obtained, for the orbit after the impulse. It is $f_{0}= \pm \cos ^{-1}\left(\frac{1}{e_{s}}\left(\frac{p}{r_{p}}-1\right)\right)$. This is the deflection angle between $\vec{r}_{p}$ and $\vec{r}_{p+}$. The value of $\alpha$ defines whether $f_{0}$ is positive or negative. If $0^{\circ}<\alpha<180^{\circ}$, the spacecraft is sent to the direction opposite to the secondary body. It will have already passed by the periapsis of the second orbit and the true anomaly $f_{0}$ is positive. If $-180^{\circ}<\alpha<0^{\circ}$, the spacecraft is sent to the direction of the secondary body and it will still pass by the periapsis of the second orbit. Therefore, the true anomaly $f_{0}$ is negative.

The true anomaly of the asymptotes of the second orbit of the spacecraft, after the impulse is applied, is given by $f_{\text {lim }}=\cos ^{-1}\left(-\frac{1}{e_{s}}\right)$. Therefore, the total deflection angle is given by $\Theta \stackrel{=}{=} \delta-f_{0}+f_{\text {lim }}-90^{\circ}$. Remember that $\delta=\sin ^{-1}\left(\frac{1}{1+\left(r_{p} V_{i n f}^{2} / \mu\right)}\right)$ (Broucke 1988). In the equations for $\Delta V$ and $\Delta E$, the term $\pm \cos ^{-1}$ takes the positive sign for $\alpha<0$ and the negative sign for $\alpha>0$. From this information, the velocity variation for a powered Swing-by maneuver with main bodies in elliptical orbits can be found. It is given by Eq. (7) shown next.

$$
\begin{aligned}
& \Delta V=\sqrt{V_{i n f-}^{2}+V_{i n f+}^{2}-2 V_{i n f-} V_{i n f+} \cos \Theta} \\
& =\left(\delta V^{2}+2 \delta V \cos \alpha \sqrt{V_{i n f-}^{2}+\frac{2 \mu}{r_{p}}}+2 V_{i n f-}^{2}-2 V_{i n f-} \sqrt{\delta V^{2}+2 \delta V \cos \alpha \sqrt{V_{i n f-}^{2}+\frac{2 \mu}{r_{p}}+V_{i n f-}^{2}}}\right. \\
& \times \sin \left(\delta+\cos ^{-1}\left(-\frac{1}{\sqrt{\frac{r_{p}\left(2 \delta V \cos \alpha r_{p} \sqrt{\left.V_{i n f-}^{2}+\frac{2 \mu}{r_{p}}+r_{p}\left(V_{i n f-}^{2}+\frac{2 \mu}{r_{p}}\right)+\delta V^{2} r_{p}-2 \mu\right)\left(\delta V \cos \alpha+\sqrt{V_{i n f-}^{2}}+\frac{2 \mu}{r_{p}}\right)^{2}}\right.}{\mu^{2}}}+1}\right)\right. \\
& \left.\left. \pm \cos ^{-1}\left(\frac{2 \delta V \cos \alpha r_{p} \sqrt{V_{i n f-}^{2}+\frac{2 \mu}{r_{p}}}+r_{p}\left(V_{i n f-}^{2}+\frac{2 \mu}{r_{p}}\right)+\delta V^{2} \cos ^{2} \alpha r_{p}-\mu}{\mu \sqrt{\frac{r_{p}\left(2 \delta V \cos \alpha r_{p} \sqrt{V_{i n f-}^{2}+\frac{2 \mu}{r_{p}}}+r_{p}\left(V_{i n f-}^{2}+\frac{2 \mu}{r_{p}}\right)+\delta V^{2} r_{p}-2 \mu\right)\left(\delta V \cos \alpha+\sqrt{V_{i n f-}^{2}+\frac{2 \mu}{r_{p}}}\right)^{2}}{\mu^{2}}}+1}\right)\right)\right)^{1 / 2}
\end{aligned}
$$

As an example, Fig. 3 shows the velocity variation as a function of the magnitude and direction of the impulse for a generic system with masses and distances similar to the Earth-Moon, but with a larger eccentricity, to show better the influence of this variable. It means a system with parameters $\mu=0.01214$ and $e=0.1$. We also used the values $v=0^{\circ}, \psi=270^{\circ}, r_{p}=1.1$ radius of the Moon (1.0 radius of the Moon is approximately $1730 \mathrm{~km}$ ) and $V_{\text {inf }-}=1.02$ $\mathrm{km} / \mathrm{s}$, to try to maximize the energy gains. The velocity of approach $\left(V_{\text {inf }}\right)$ used in the present research is above the minimum possible value for a spacecraft to reach the Moon coming from a low orbit near Earth using a Hohmann type maneuver. This margin is used here to take into account the variations of the velocity of Moon in its orbit around the Earth and the fact that it is common to use a transfer that is faster than the Hohmann type. 
Fig. 3 Velocity variation (in $\mathrm{km} / \mathrm{s}$ ) due to the powered Swing-By maneuver for a system with $\mu=0.01214$, $e=0.1, v=0^{\circ}, \psi=270^{\circ}$, $r_{p}=1.1$ radius of $M_{2}$ and $V_{\text {inf- }}=1.02 \mathrm{~km} / \mathrm{s}$. Plot generated from Eq. (7)

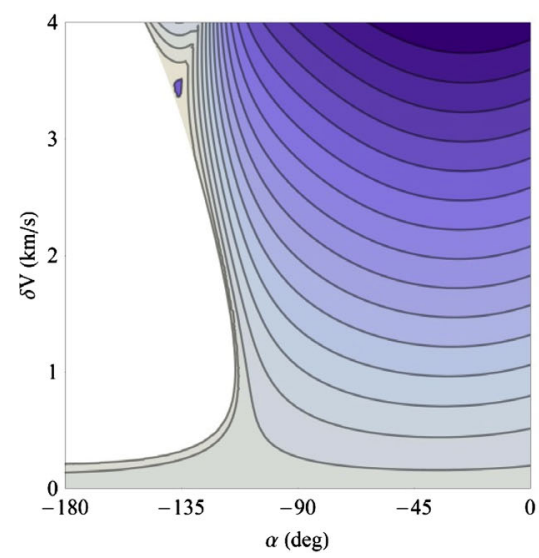

(a) $\alpha<0$

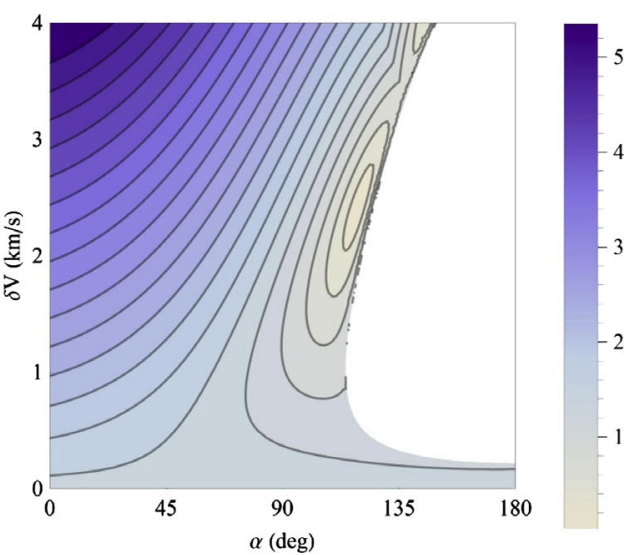

(b) $\alpha>0$
Figure 3(a) shows and quantifies the increase of the velocity with the magnitude of the impulse when the maneuver sends the spacecraft to the direction of $M_{2}$. In this case, the maximum variations are near $\alpha=-20^{\circ}$. This is a maneuver with gains of energy $\left(\psi=270^{\circ}\right)$, so sending the spacecraft to the direction of $M_{2}$ increases the effect of the gravity part of the maneuver, contributing to maximize the total energy variation. This gain compensates the loss of energy due to the fact that the impulse is not applied in the direction of motion of the spacecraft. This is in agreement with Prado (1996) that studied the circular case. Figure 3(b) shows the opposite behavior, when the spacecraft is directed to the opposite direction of $M_{2}$. In this situation, the energy variation decreases with the angle of the impulse, because the spacecraft goes away from $M_{2}$, so reducing the energy gains from the gravity part of the maneuver. Combined with the losses due to the non alignment of impulse and velocity, the net result is a decrease in the energy variation. In both cases the maximum velocity variations have components in the direc- tion of the motion of the spacecraft. Those variations are independent of the eccentricity and the location of $M_{2}$ in its orbit, as shown by Eq. (7). In both figures the blank regions indicates the locations of captures or collisions.

The energy variation is given by $\Delta E=\frac{1}{2}\left(\vec{V}_{o}^{2}-\vec{V}_{i}^{2}\right)$ or $\Delta E=\vec{V}_{2} . \Delta \vec{V}$ (Broucke 1988; Barger and Olsson 1973). It is written as a function of the three independent parameters related to the pure gravity Swing-By and the parameters related to the eccentricity of the orbit of the two main bodies. Consider $\beta=\cos ^{-1}\left(-\frac{V_{r}}{V_{2}}\right) ; V_{r}=e \sqrt{\frac{(1-\mu)}{a\left(1-e^{2}\right)}} \sin v$ the radial velocity of the secondary body; $V_{2}=\sqrt{(1-\mu)\left(\frac{2}{d}-\frac{1}{a}\right)}$; $a$ the semi-major axis of the orbit of the two main bodies; $e$ the eccentricity of this orbit; $v$ the true anomaly of $M_{2}$ in its orbit around $M_{1}$ when the close approach occurs and $d=\frac{a\left(1-e^{2}\right)}{1+e \cos v}$ the distance between the two main bodies at this moment. Then, Eq. (8) shows the energy variation given by this maneuver.

$$
\begin{aligned}
& \Delta E=\frac{1}{2}\left(V_{i n f+}^{2}-V_{i n f-}^{2}-2 V_{2} V_{i n f-} \sin (\beta-\delta+\psi)+2 V_{2} V_{i n f+} \sin (\beta-\delta+\Theta+\psi)\right)
\end{aligned}
$$

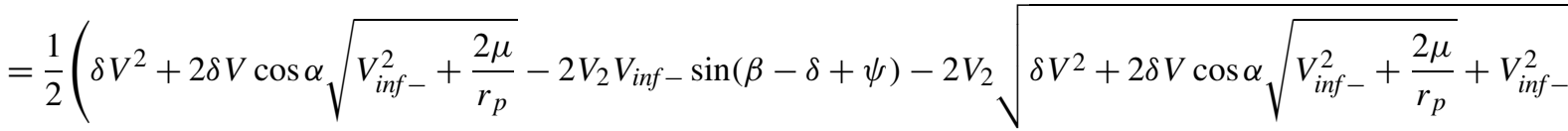

$$
\begin{aligned}
& \times \cos \left(\beta+\psi \pm \cos ^{-1}\left(\frac{\frac{r_{p}\left(-2 \delta V \cos \alpha \sqrt{V_{i n f-}^{2}+\frac{2 \mu}{r_{p}}}-2 V_{i n f-}^{2}-\frac{4 \mu}{r_{p}}\right)^{2}}{4 \mu\left(V_{i n f-}^{2}+\frac{2 \mu}{r_{p}}\right)}-1}{\sqrt{\frac{r_{p}^{2}\left(\delta V^{2}+2 \delta V \cos \alpha \sqrt{V_{i n f-}^{2}+\frac{2 \mu}{r_{p}}}+V_{i n f-}^{2}\right)\left(-2 \delta V \cos \alpha \sqrt{V_{i n f-}^{2}+\frac{2 \mu}{r_{p}}}-2 V_{i n f-}^{2}-\frac{4 \mu}{r_{p}}\right)^{2}}{4 \mu^{2}\left(V_{i n f-}^{2}+\frac{2 \mu}{r_{p}}\right)}+1}}\right)\right.
\end{aligned}
$$

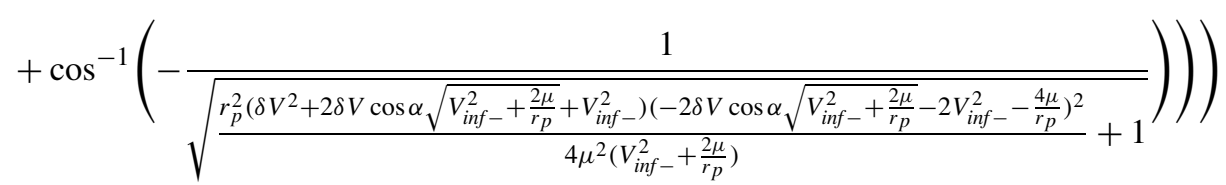




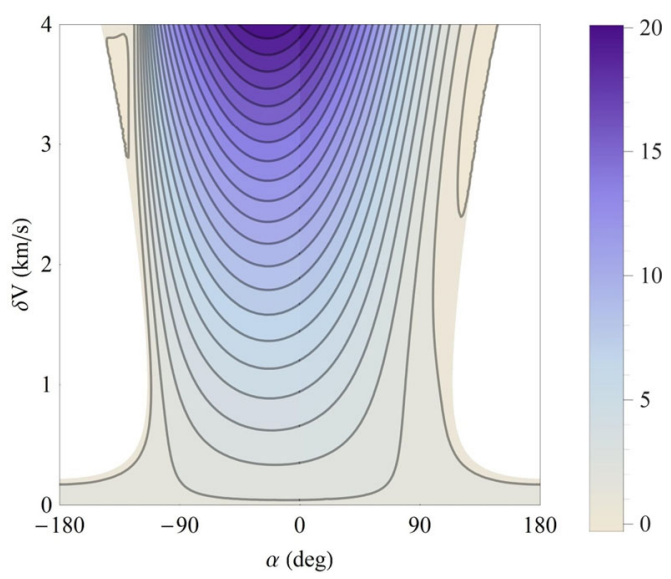

Fig. 4 Energy variation (in $\mathrm{km}^{2} / \mathrm{s}^{2}$ ) due to the powered Swing-By maneuver for a system with $\mu=0.01214, e=0.1, v=0^{\circ}, \psi=270^{\circ}$, $r_{p}=1.1$ radius of $M_{2}$ and $V_{i n f-}=1.02 \mathrm{~km} / \mathrm{s}$. Plot generated from Eq. (8)

As an example, Fig. 4 shows the energy variation for the same system used before. It means that $\mu=0.01214$, $e=0.1, v=0^{\circ}, \psi=270^{\circ}, r_{p}=1.1$ radius of $M_{2}$ and $V_{\text {inf }-}=1.02 \mathrm{~km} / \mathrm{s}$. In this case $\beta=90^{\circ}$, because the secondary body is in the periapsis of its orbit around the primary. Note that the energy variations increase with the magnitude of the impulse and the best directions to apply the impulse to maximize the energy variations are around $\alpha=$ $-20^{\circ}$. It happens because $\psi=270^{\circ}$ is the region of energy gains due to the gravity part of the maneuver, so impulses with negative values of $\alpha$ send the spacecraft to the direction of the secondary body, therefore maximizing the effect of gravity, as already explained above, and similar to which occurs in the circular case (Prado 1996). Observe the positive values of the variations.

If the impulse is zero, the energy variation dependents only on the gravity effect and the equation for the variation of energy reduces to $\Delta E=2 V_{\text {inf }}-V_{2} \sin \delta \cos (\psi+\beta)$, which is equivalent to the pure gravity Swing-By in an elliptic system (Ferreira et al. 2018).

The blank regions near the borders of the plots also represent captures of the spacecraft by the secondary body or collisions with the same body. From the analytical equations, it is only possible to identify the situations where the spacecraft did not escape from the region of influence of the secondary body. It is not possible to distinguish between captures and collisions. This distinction is only possible to be made from a numerical study.

Next, a study is made to show the effect of the eccentricity and the true anomaly of the secondary body at the moment of the close approach in the energy variation, always using $\alpha=0^{\circ}$ to reduce the number of variables in the problem and with the magnitude of the impulse assuming the values $\delta V=0$ (no impulse) and $\delta V=0.5 \mathrm{~km} / \mathrm{s}$. The same data for the system is used, except for the eccentricity,

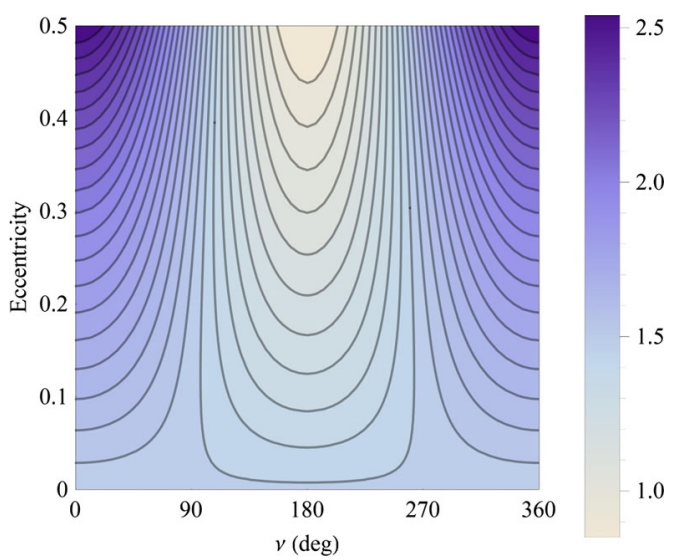

Fig. 5 Energy variation (in $\mathrm{km}^{2} / \mathrm{s}^{2}$ ) due to the powered Swing-By maneuver for a system with $\mu=0.01214, \psi=270^{\circ}, r_{p}=1.1$ radius of $M_{2}$ and $V_{\text {inf- }}=1.02 \mathrm{~km} / \mathrm{s}$ as a function of $e$ and $v$ for $\delta V=0$. Plot generated from Eq. (8)

which is varied in the range 0 to 0.5 to show the effects of this parameter.

Note that, for zero impulse, the variations depend only on the gravitational part of the maneuver. Figure 5 shows that the largest variations of energy occur near the periapsis of the orbit $\left(v=0^{\circ}\right.$ or $\left.v=360^{\circ}\right)$. This is due to the velocity of the secondary body around the primary, which is a parameter in the equation for the energy variation $(\Delta E=$ $2 V_{\text {inf }}-V_{2} \sin \delta \cos (\psi+\beta)$, Ferreira et al. 2018). At those locations, the energy variation increases with the eccentricity, because this increase generates higher velocities at periapsis for a fixed true anomaly. The opposite occurs for true anomalies near the apoapsis. At this location the orbital velocity is minimum, which reduces the energy variation. Therefore, at this location, the increase of the eccentricity decreases the variation of energy, because lower eccentricities generate higher apoapsis velocities.

Figure 6 shows the variations of energy when $\delta V=$ $0.5 \mathrm{~km} / \mathrm{s}$, applied in the direction of the movement of the spacecraft. The magnitude of the energy is higher in this case due to the contribution of the impulse. It is also noted that there is a shift in the maximum variation of energy with respect to the true anomaly of $M_{2}$, caused by the application of the impulsive maneuver. The maximum values are no longer in $v=0^{\circ}$, but in values close to $30^{\circ}$. More specific equations are developed next to show this fact in detail. It is a consequence of the complex dynamics of the system and it is shown in the present paper for the first time. The velocity $V_{2}$ is smaller, which reduces the variations of energy, but the direction of the impulse also changes ( since $\alpha=0^{\circ}$ ), so giving different effects in the maneuver. The net result of those effects is the shift in the maximum variations of energy.

Applying some of the values used in the simulations, it is possible to get an equation of $\Delta E$ as function only of $v$ and $\delta V$, which helps to explain the shift in the maximum 
variations of energy. It is shown in Eq. (9), where it is used the values $\mu=0.01214, \psi=270^{\circ}, r_{p}=1.1$ radius of $M_{2}$, $e=0.1$ and $V_{\text {inf }-}=1.02 \mathrm{~km} / \mathrm{s}$. Figure 7 shows Eq. (9) in a graphical format. It is clearly noted and quantified the in- creases of the energy variations with the magnitude of the impulse and the oscillations with the true anomaly of the secondary body. The shift in the maximum variation of energy is also clearly shown.

$$
\begin{aligned}
\Delta E= & 0.5\left(-0.1998 \sqrt{\delta V^{2}+4.86 \delta V+1} \cos \left(-\cos ^{-1}\left(\frac{04077 \delta V^{2}+1.9817 \delta V+1.4077}{\sqrt{0.1663\left(\delta V^{2}+4.86 \delta V+1\right)(\delta V+2.43)^{2}+1}}\right)\right.\right. \\
& \left.+\cos ^{-1}\left(-\frac{1}{\sqrt{0.1663\left(\delta V^{2}+4.86 \delta V+1\right)(1 \delta V+2.43)^{2}+1}}\right)+v\right)+\delta V^{2} \\
& -1.998 \sqrt{\delta V^{2}+4.86 \delta V+1} \cos \left(\cos ^{-1}\left(-\frac{1}{\sqrt{0.1663\left(\delta V^{2}+4.86 \delta V+1\right)(1 \delta V+2.43)^{2}+1}}\right)\right. \\
& \left.\left.-\cos ^{-1}\left(\frac{0.4077 \delta V^{2}+1.9817 \delta V+1.4077}{\sqrt{0.1663\left(\delta V^{2}+4.86 \delta V+1\right)(\delta V+2.43)^{2}+1}}\right)\right)+4.86 \delta V-0.1998 \cos (v+3.9224)+1.4192\right)
\end{aligned}
$$

To show better the shift discovered here, a new equation is developed using a magnitude of the impulse of $0.5 \mathrm{~km} / \mathrm{s}$, such that it is possible to visualize the variations of energy $\Delta E$ as function only of the true anomaly $\nu$. It is shown in Eq. (10), that uses the parameters: with $\mu=0.01214$, $\psi=270^{\circ}, r_{p}=1.1$ radius of $M_{2}, e=0.1 V_{\text {inf }-}=1.02 \mathrm{~km} / \mathrm{s}$ and $\delta V=0.5 \mathrm{~km} / \mathrm{s}$. The graphical representation is shown in Fig. 8. Equation (10) and Fig. 8 gives a clear view of the shift of the maximum energy variation with respect to the true anomaly of $M_{2}$.

$$
\begin{aligned}
\Delta E= & 0.1916 \sin (v+6.6949)-0.0999 \cos (v+3.9224) \\
& +2.816
\end{aligned}
$$

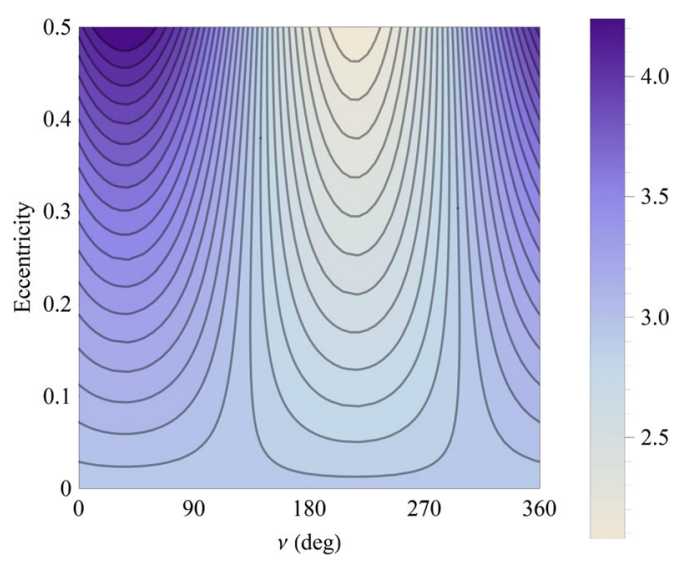

Fig. 6 Energy variation (in $\mathrm{km}^{2} / \mathrm{s}^{2}$ ) due to the powered Swing-By maneuver for a system with $\mu=0.01214, \psi=270^{\circ}, r_{p}=1.1$ radius of $M_{2}$ and $V_{\text {inf- }}=1.02 \mathrm{~km} / \mathrm{s}$ as a function of $e$ and $v$ for $\delta V=0.5 \mathrm{~km} / \mathrm{s}$. Plot generated from Eq. (8)

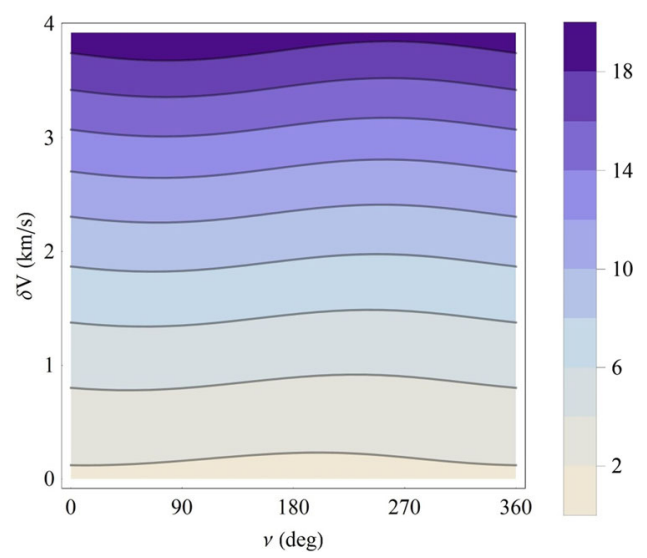

Fig. 7 Energy variation (in $\mathrm{km}^{2} / \mathrm{s}^{2}$ ) due to the powered Swing-By maneuver for a system with $\mu=0.01214, \psi=270^{\circ}, r_{p}=1.1$ radius of $M_{2}, e=0.1$ and $V_{\text {inf- }}=1.02 \mathrm{~km} / \mathrm{s}$ as a function of $v$ and $\delta V$. Plot generated from Eq. (9)

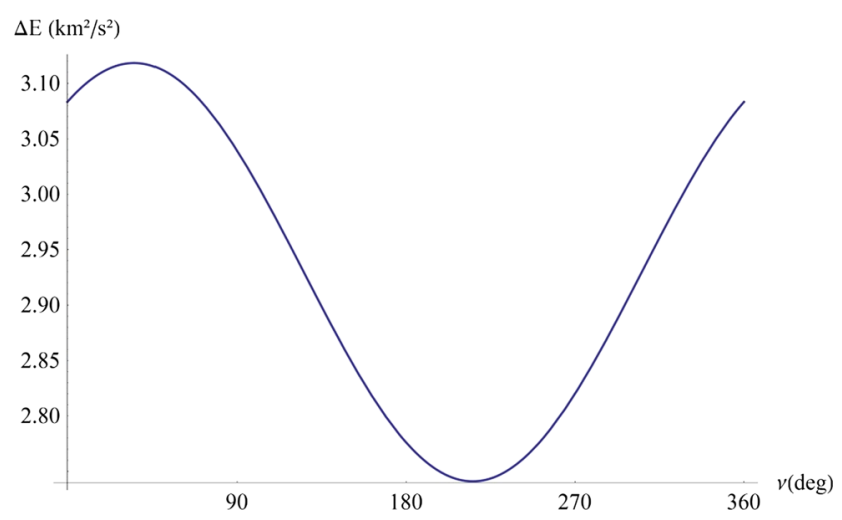

Fig. 8 Energy variation (in $\mathrm{km}^{2} / \mathrm{s}^{2}$ ) due to the powered Swing-By maneuver for a system with $\mu=0.01214, \psi=270^{\circ}, r_{p}=1.1$ radius of $M_{2}, V_{\text {inf- }}=1.02 \mathrm{~km} / \mathrm{s}, e=0.1$ and $\delta V=0.5 \mathrm{~km} / \mathrm{s}$, as a function of $\nu$. Plot generated from Eq. (10) 


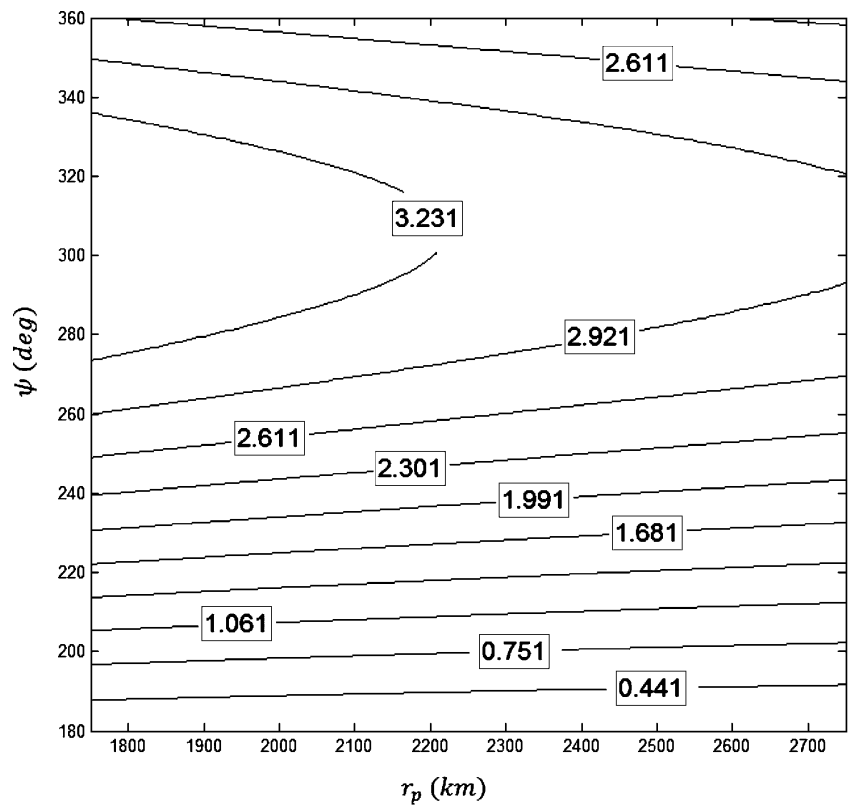

(a) $\Delta E_{\text {imp }}$ using $V_{\text {inf- }}=1.02 \mathrm{~km} / \mathrm{s}$

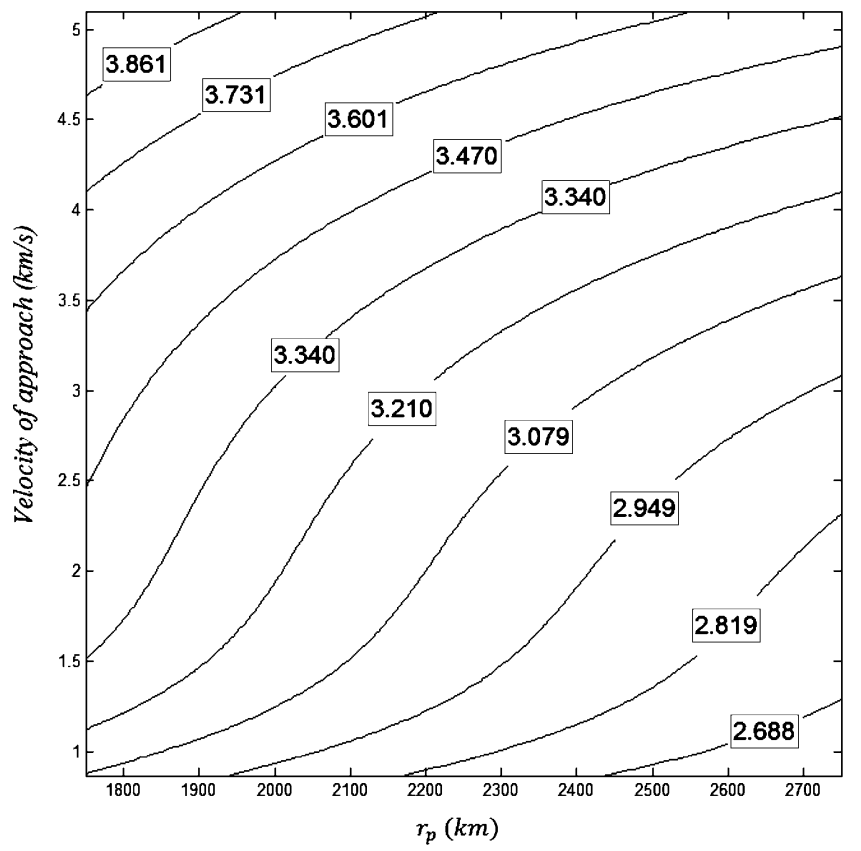

(c) $\Delta E_{\text {imp }}$ using $\psi=270^{\circ}$

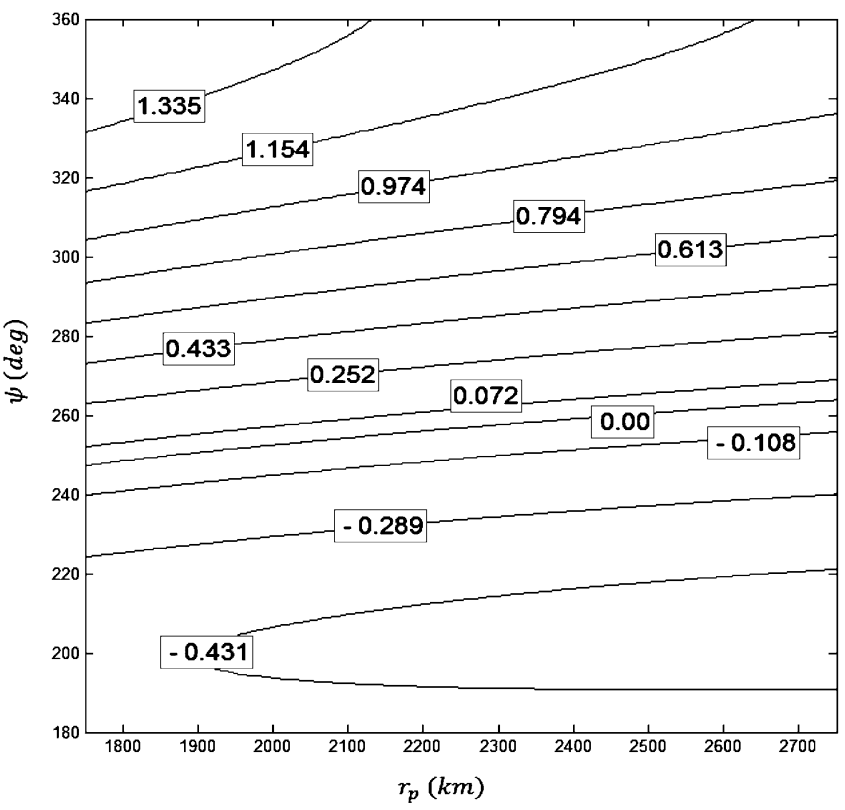

(b) Efficiency using $V_{\text {inf- }}=1.02 \mathrm{~km} / \mathrm{s}$

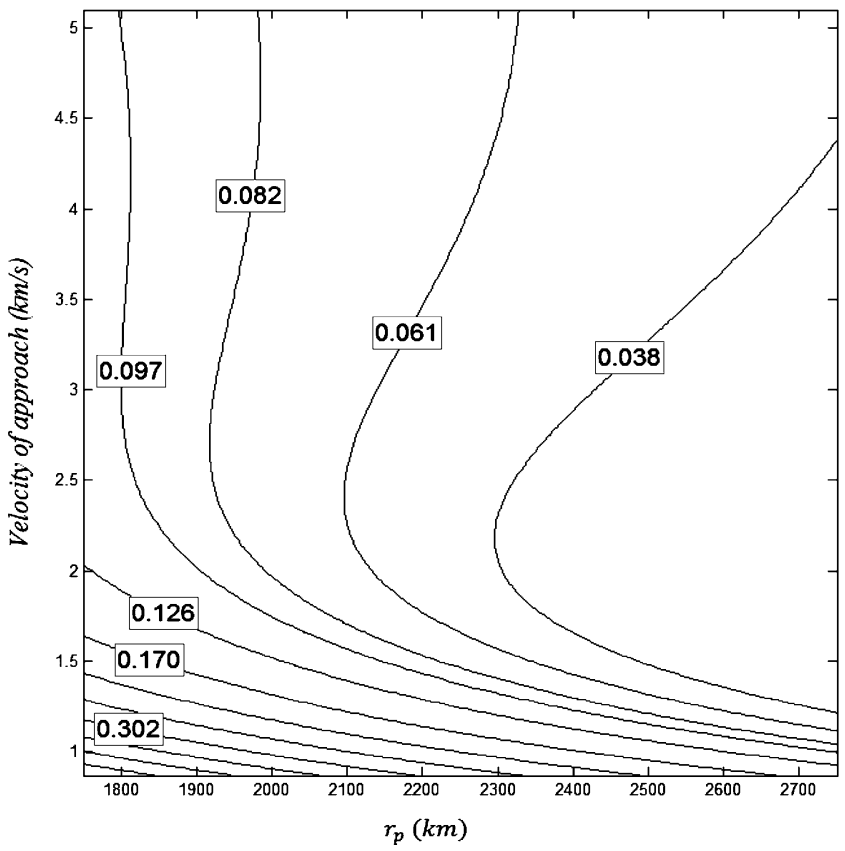

(d) Efficiency using $\psi=270^{\circ}$

Fig. 9 Analysis of the energy variation and efficiency in the energy gains region of the powered Swing-By maneuver for a system with $\mu=0.01214, e=0.1, v=0^{\circ}$ and $\delta V=0.5 \mathrm{~km} / \mathrm{s}$

\section{Powered Swing-By efficiency}

The efficiency of the powered Swing-By is defined as the amount of extra energy that is obtained by the spacecraft when the impulse is applied in the moment of the closest approach, when compared to the maneuver where an impulse, with the same magnitude, is applied after the spacecraft leaves the sphere of influence of $M_{2}$.
First, it is calculated the variation of energy $\Delta E_{\text {imp }}$ obtained from the Swing-By maneuver with the impulse applied in the periapsis of the orbit. Then, the energy variation $\Delta E_{\text {after }}$ of the maneuver with the impulse applied outside the sphere of influence of the secondary body is obtained. This maneuver is divided in two steps: (i) a pure gravity Swing-By, without the application of the impulse, with the 


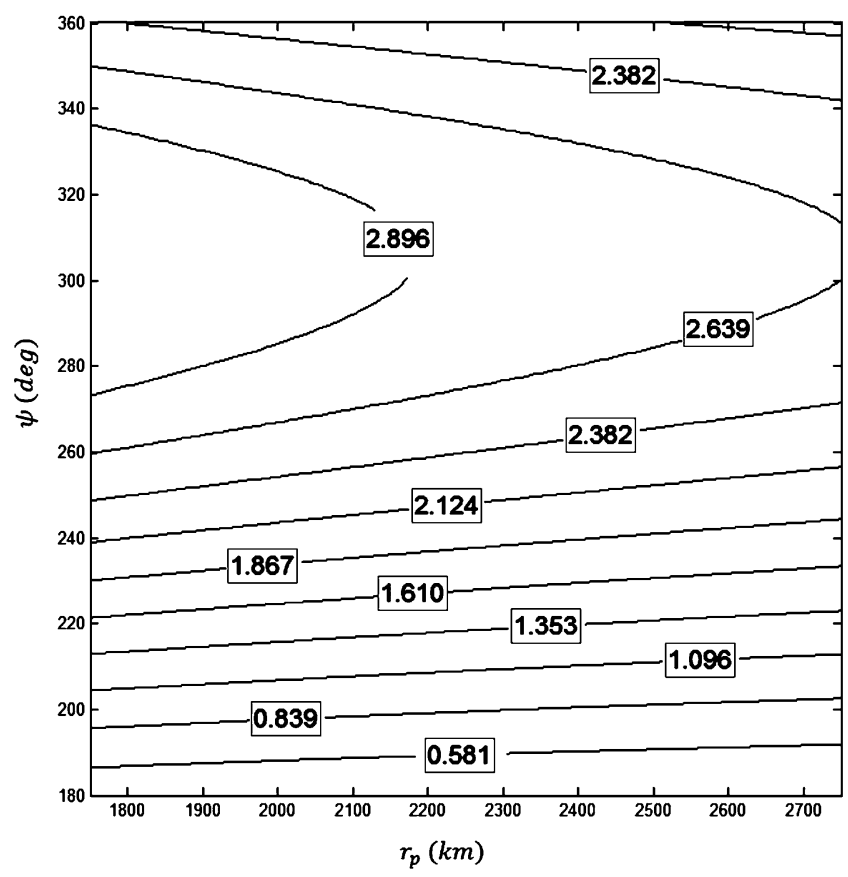

(a) $\Delta E_{\text {imp }}$ using $V_{\text {inf- }}=1.02 \mathrm{~km} / \mathrm{s}$

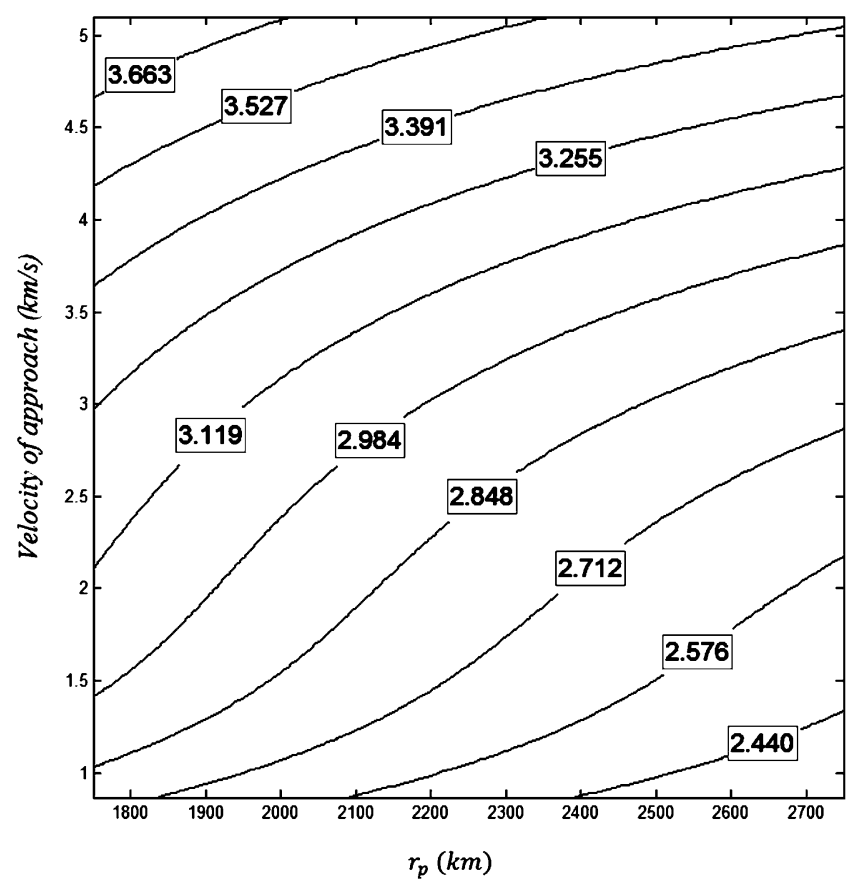

(c) $\Delta E_{\text {imp }}$ using $\psi=270^{\circ}$

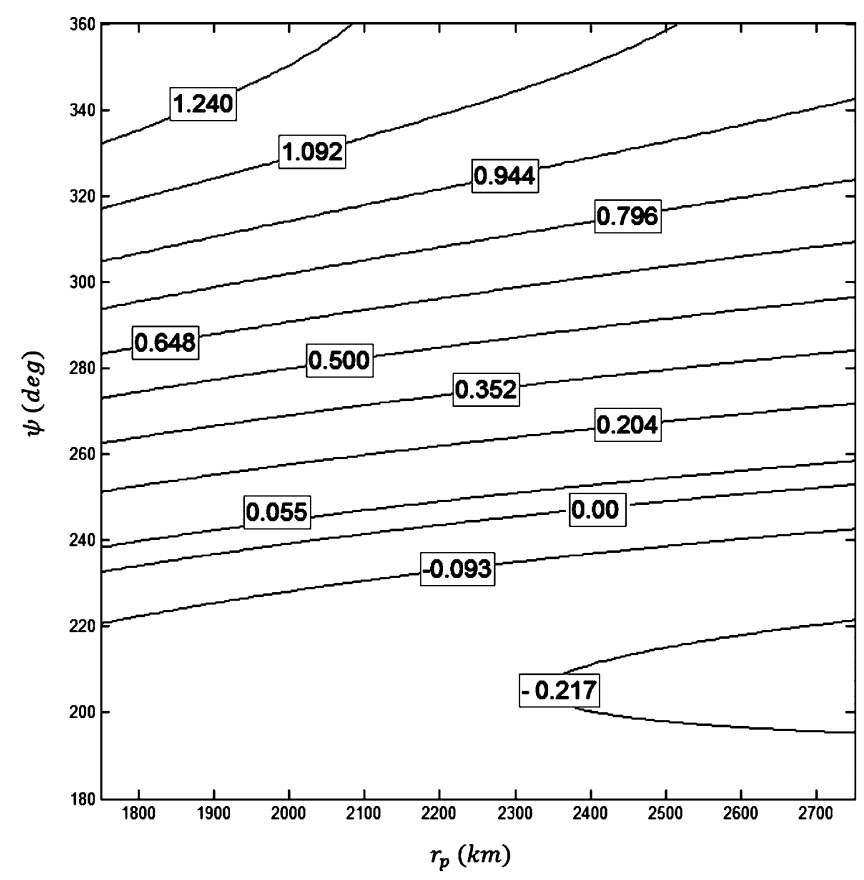

(b) Efficiency using $V_{\text {inf- }}=1.02 \mathrm{~km} / \mathrm{s}$

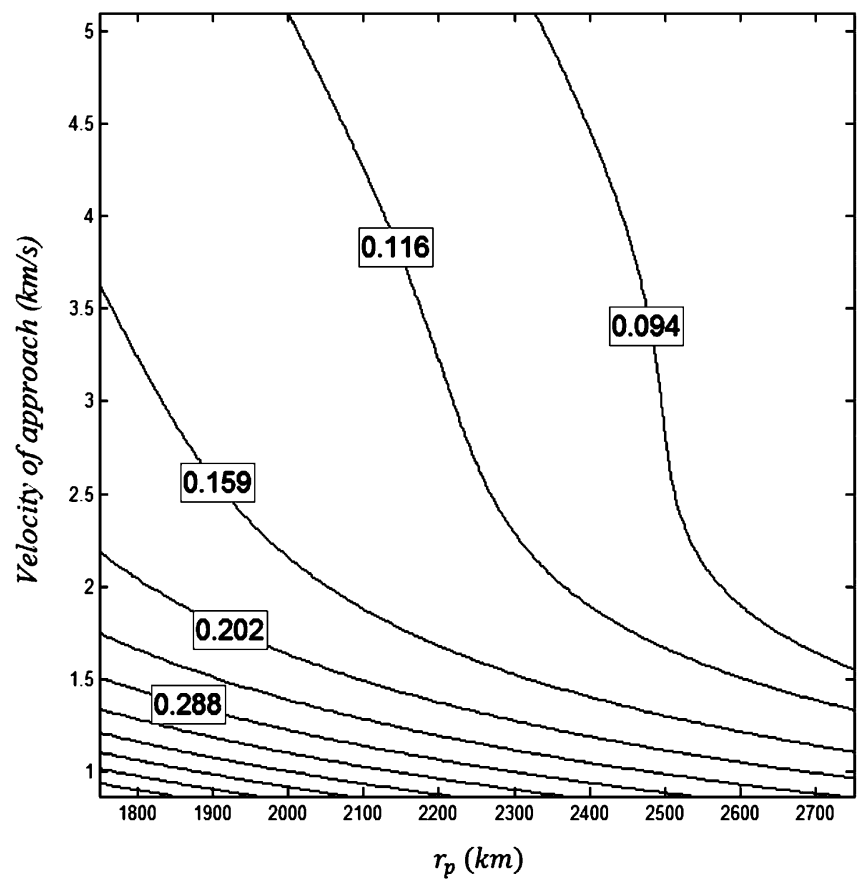

(d) Efficiency using $\psi=270^{\circ}$

Fig. 10 Analysis of the energy variation and efficiency in the energy gains region of the powered Swing-By for a system with $\mu=0.01214$, $e=0.1, v=180^{\circ}$ and $\delta V=0.5 \mathrm{~km} / \mathrm{s}$

same parameters of the powered Swing-By and, after that: (ii) the application of an impulse with the same magnitude of the powered Swing-By maneuver after the spacecraft leaves the sphere of influence of the secondary body.

In the energy gain region, $-90^{\circ}<\psi+\beta<90^{\circ}$, the impulse is applied in the direction of the motion of the space- craft, so $\alpha=0^{\circ}$, to maximize the transfer of energy coming from the impulse, since the goal of the maneuver is to increase the energy. In the energy loss region, $90^{\circ}<\psi+\beta<$ $270^{\circ}$, the impulse is applied in the direction opposite to the motion of the spacecraft, so $\alpha=180^{\circ}$, to maximize the energy removal due to the impulse, which is the goal of the 


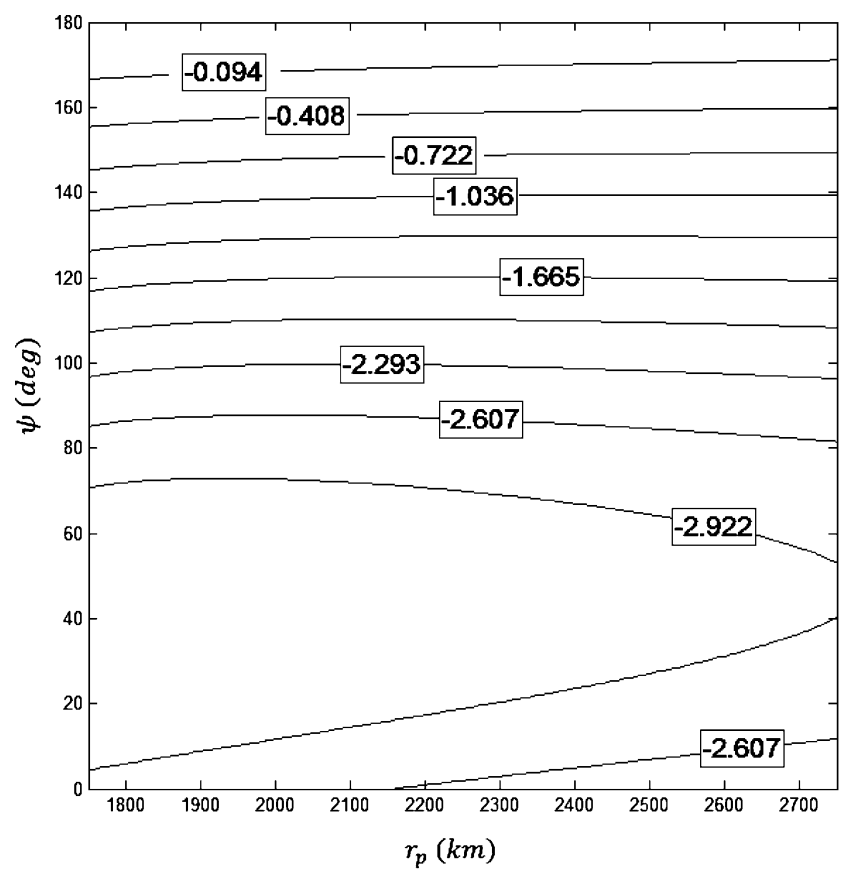

(a) $\Delta E_{\text {imp }}$ using $V_{\text {inf- }}=1.6 \mathrm{~km} / \mathrm{s}$

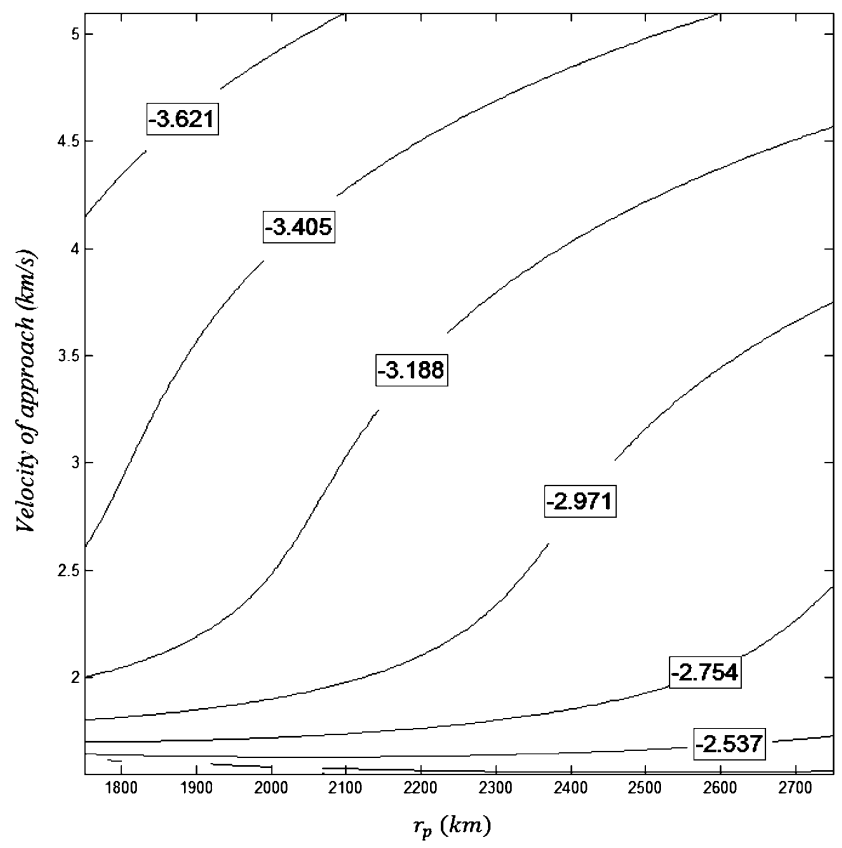

(c) $\Delta E_{\text {imp }}$ using $\psi=90^{\circ}$

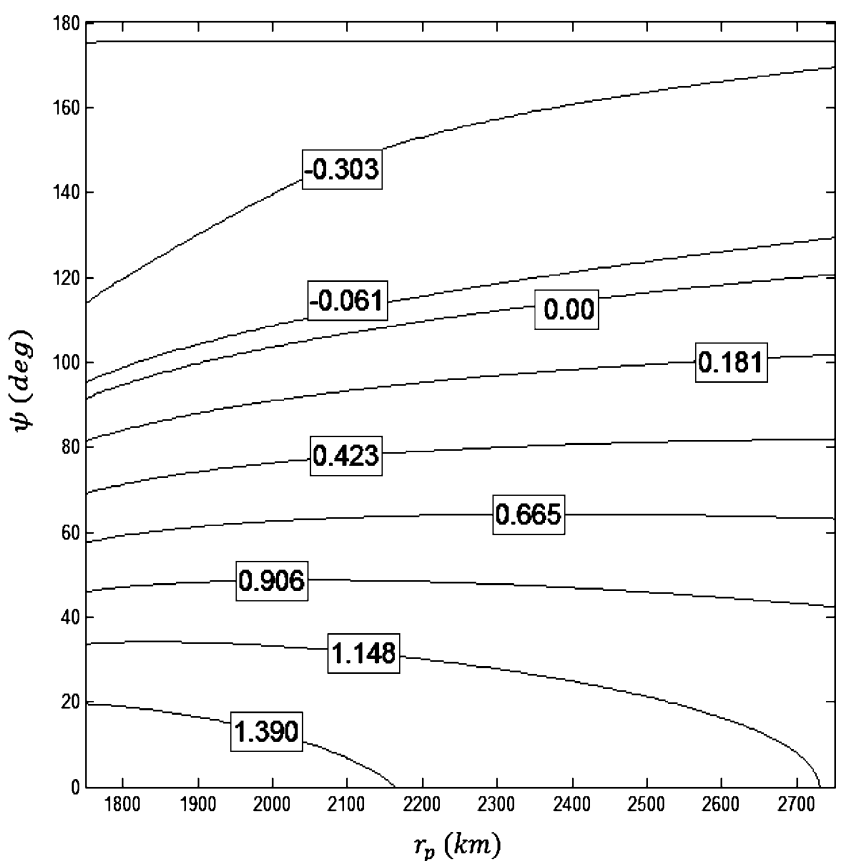

(b) Efficiency using $V_{\text {inf- }}=1.6 \mathrm{~km} / \mathrm{s}$

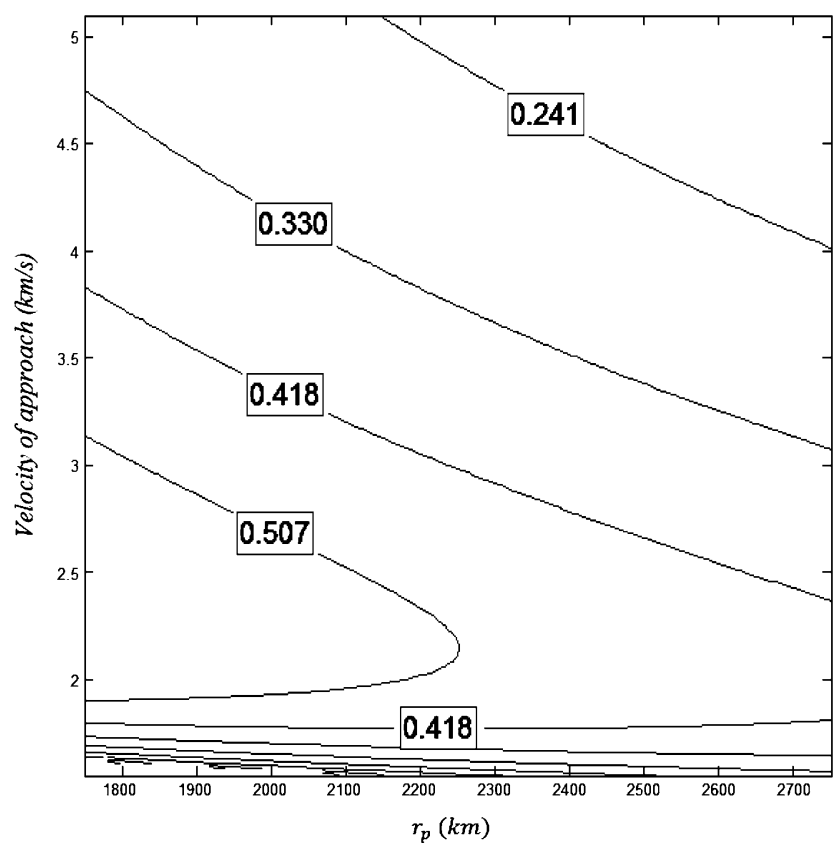

(d) Efficiency using $\psi=90^{\circ}$

Fig. 11 Analysis of the energy variation and efficiency in the energy losses region of the powered Swing-By for a generic system with $\mu=0.01214$, $e=0.1, v=0^{\circ}$ and $\delta V=0.5 \mathrm{~km} / \mathrm{s}$

maneuver. Equation (11) represents the energy gain situation and Eq. (12) the energy loss situation.

$$
\begin{aligned}
& \Delta E_{\text {after }}=\frac{1}{2}\left(V_{o}+\delta V\right)^{2}-\frac{(1-\mu)}{d}-E_{i} \\
& \Delta E_{a f t e r}=\frac{1}{2}\left(V_{o}-\delta V\right)^{2}-\frac{(1-\mu)}{d}-E_{i}
\end{aligned}
$$

In Eqs. (11)-(12), $d$ is the distance between the two main bodies, $E_{i}=\frac{1}{2}\left(V_{i X}^{2}+V_{i Y}^{2}\right)-\frac{(1-\mu)}{d}$ is the energy before the close encounter and $E_{o}=E_{i}+\Delta E$ is the energy after the close encounter. $\Delta E=2 V_{\text {inf }-} V_{2} \sin \delta \cos (\psi+\beta)$ is the energy variation due to the gravity part of the maneuver (Fer- 


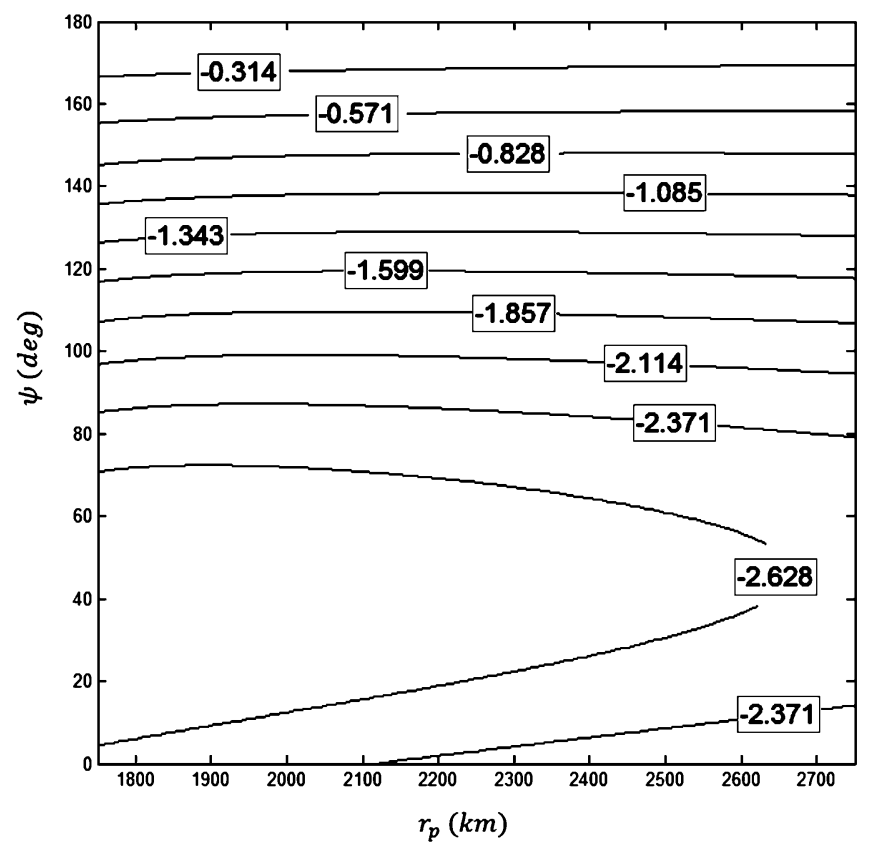

(a) $\Delta E_{\text {imp }}$ using $V_{\text {inf }-}=1.6 \mathrm{~km} / \mathrm{s}$

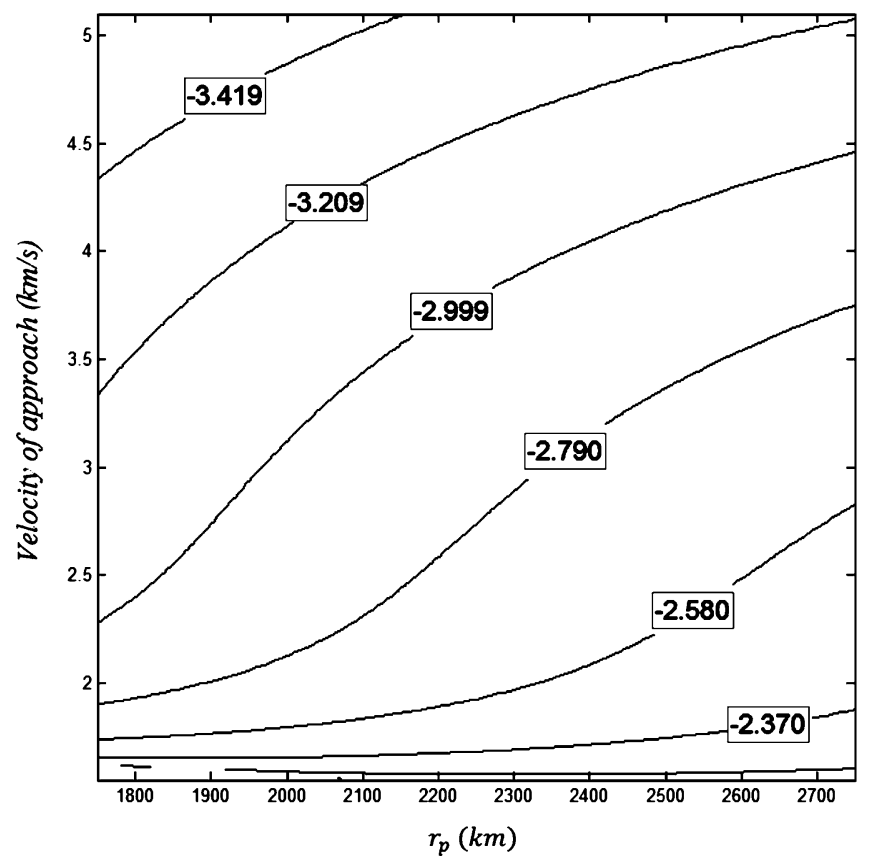

(c) $\Delta E_{\text {imp }}$ using $\psi=90^{\circ}$

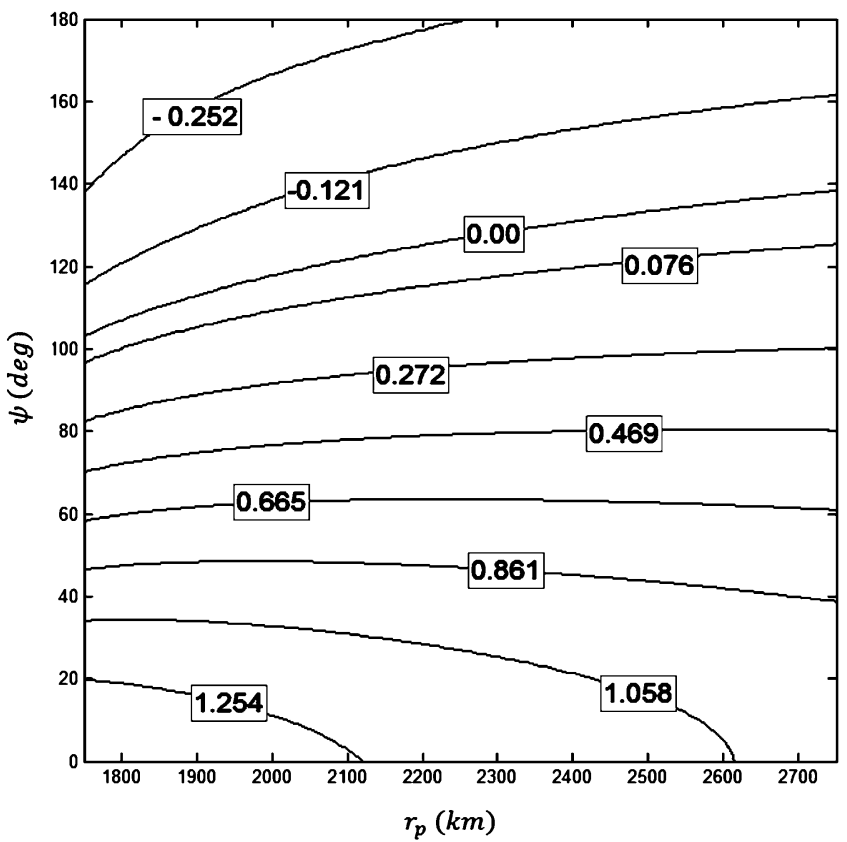

(b) Efficiency using $V_{\text {inf- }}=1.6 \mathrm{~km} / \mathrm{s}$

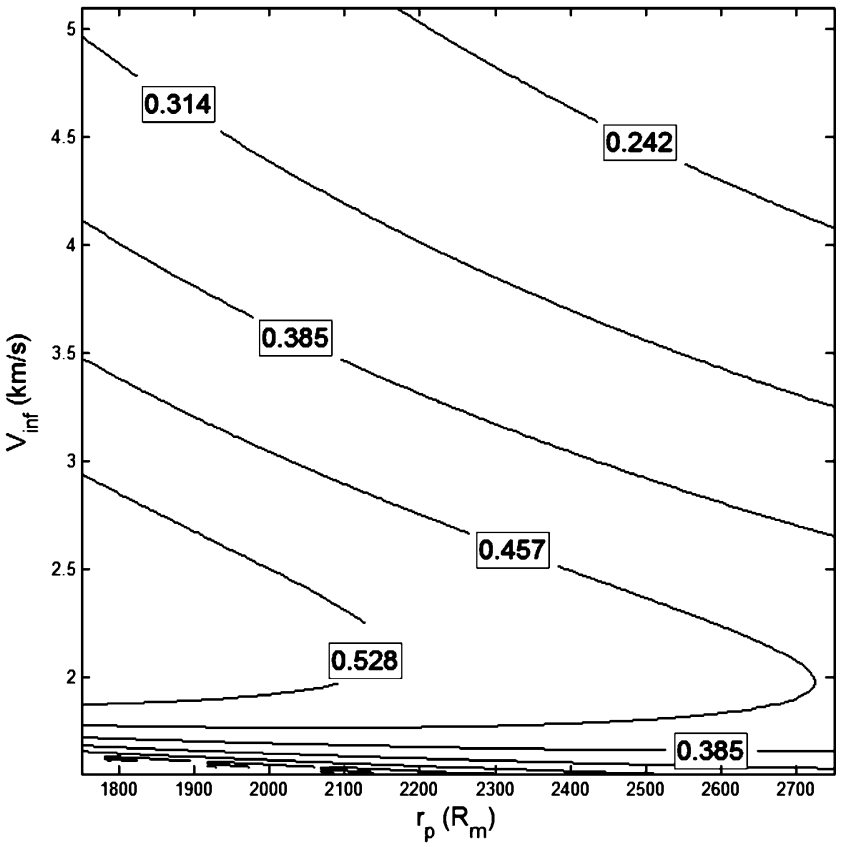

(d) Efficiency using $\psi=90^{\circ}$

Fig. 12 Analysis of the energy variation and efficiency in the energy losses region of the powered Swing-By for a generic system with $\mu=0.01214$, $e=0.1, v=180^{\circ}$ and $\delta V=0.5 \mathrm{~km} / \mathrm{s}$

reira et al. 2018) and $V_{0}=\sqrt{2\left(E_{o}+\frac{(1-\mu)}{d}\right)}$ is the velocity of the spacecraft when leaving $M_{2}$.

The efficiency of the powered Swing-By $(E f)$ is measured by $\left|\Delta E_{\text {imp }}\right|-\left|\Delta E_{\text {after }}\right|$, which is given by Eq. (13).

$E f=\mid \frac{1}{2}\left(V_{i n f+}^{2}-V_{i n f-}^{2}-2 V_{2} V_{i n f-} \sin (\beta-\delta+\psi)\right.$

$$
\begin{aligned}
& \left.+2 V_{2} V_{i n f+} \sin (\beta-\delta+\Theta+\psi)\right)|-| \delta V\left(\frac{1}{2} \delta V\right. \\
& \left. \pm \sqrt{V_{2}^{2}+V_{i n f-}^{2}+2 V_{2} V_{i n f-} \sin (\beta+\delta+\psi)}\right) \\
& +2 V_{2} V_{i n f-\cos (\beta+\psi) \sin (\delta)} \mid
\end{aligned}
$$


Fig. 13 Analysis of the energy variation and efficiency in the energy gains region of the powered Swing-By for a system with $e=0.1, v=0^{\circ}, r_{p}=1.1$ radius of $M_{2}$ and $\delta V=0.5 \mathrm{~km} / \mathrm{s}$

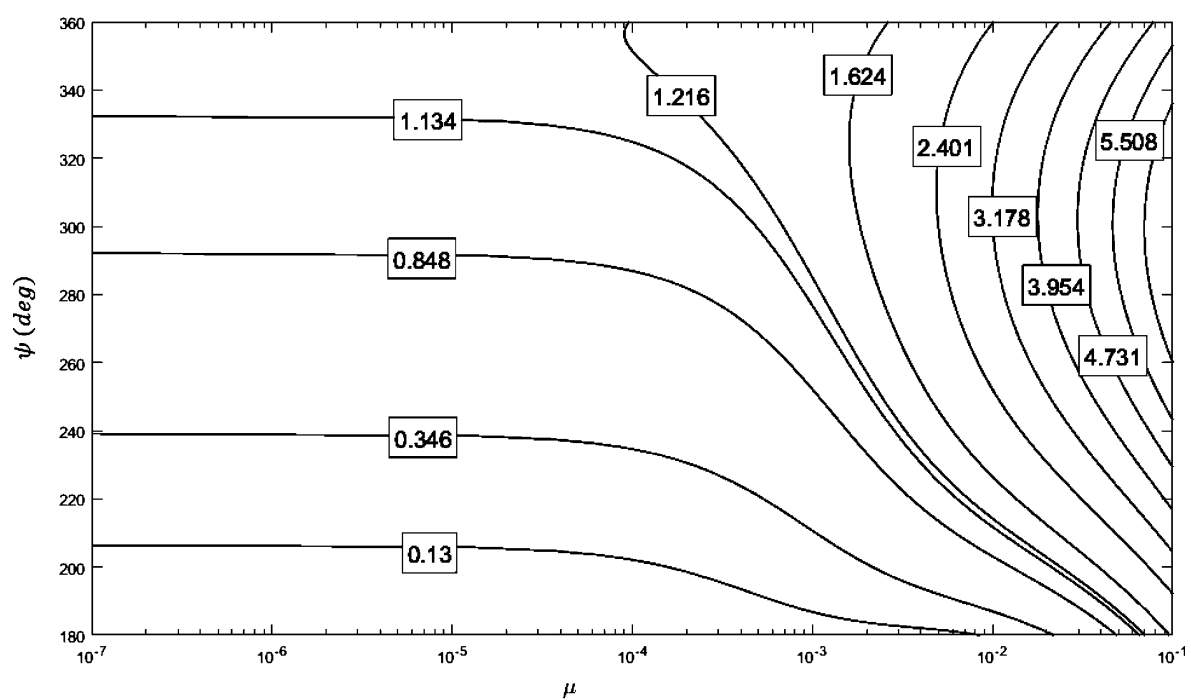

(a) $\Delta E_{\text {imp }}$ using $V_{\text {inf- }}=1.02 \mathrm{~km} / \mathrm{s}$

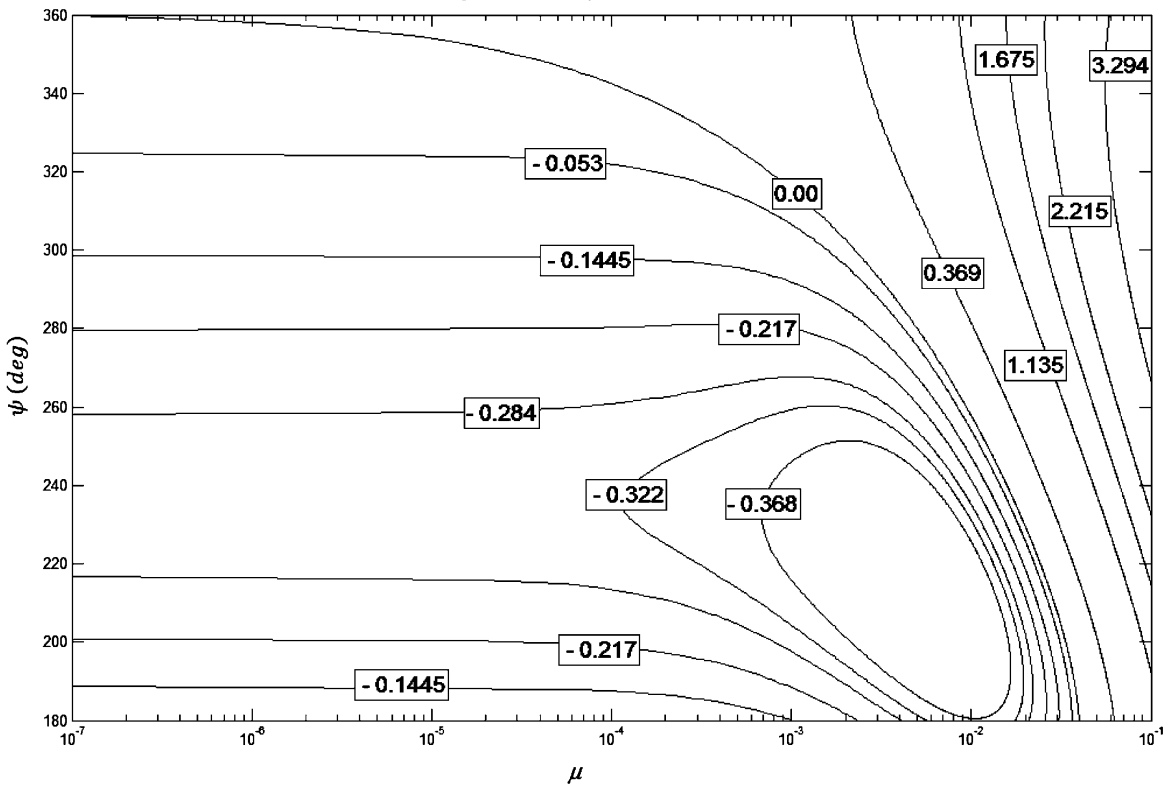

(b) Efficiency using $V_{\text {inf }-}=1.02 \mathrm{~km} / \mathrm{s}$

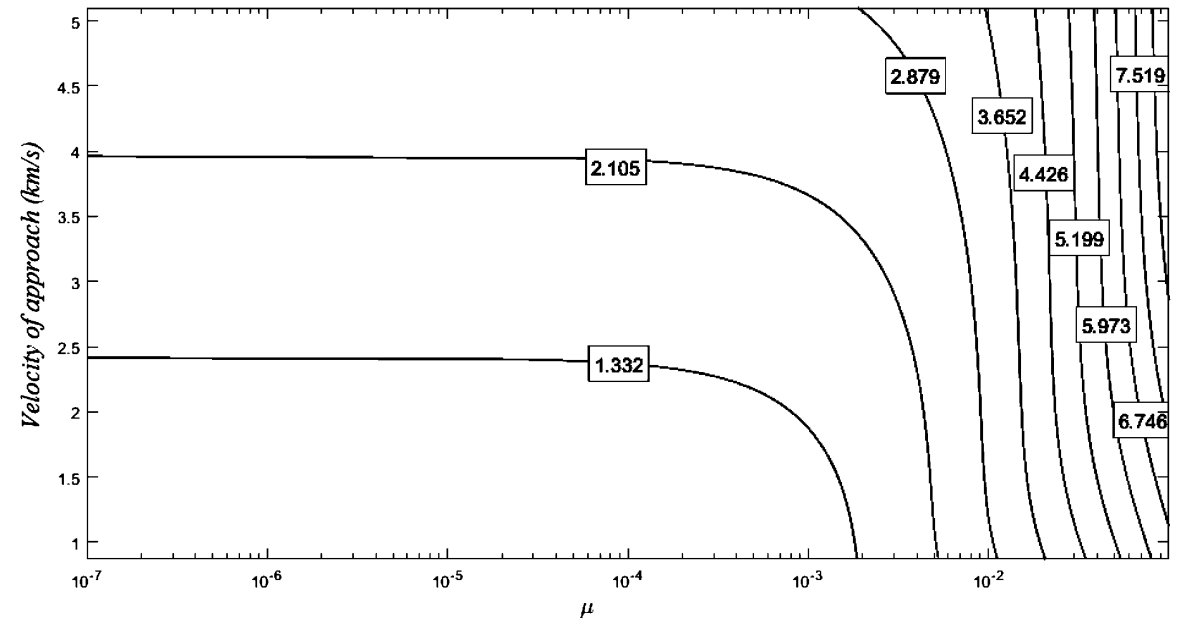

(c) $\Delta E_{\text {imp }}$ using $\psi=270^{\circ}$ 
Fig. 13 (Continued)

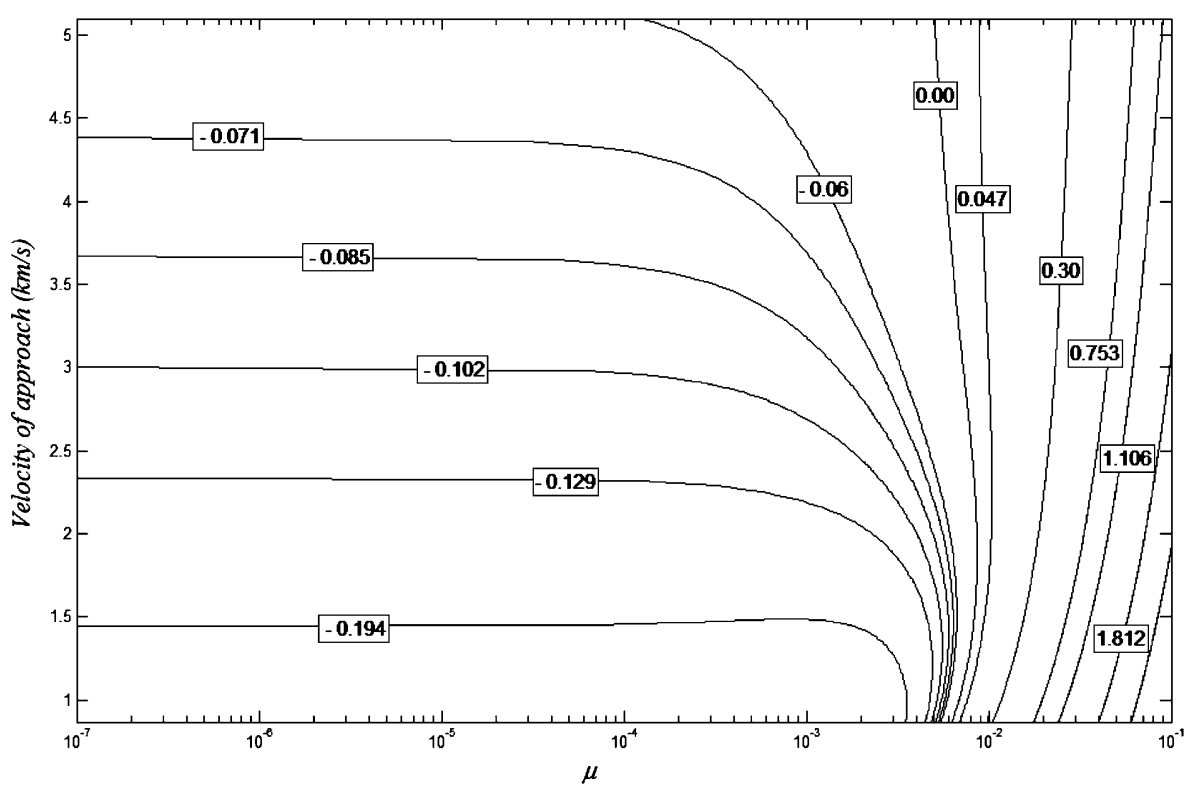

(d) Efficiency using $\psi=270^{\circ}$

If Eq. (13) gives a positive value, the application of the impulse in the periapsis of the orbit of the spacecraft around $M_{2}$ is more efficient than the application outside the sphere of influence of $M_{2}$. Otherwise, the application of the impulse at the periapsis is not the best choice.

The numerical results presented here are valid for a system with masses and distances similar to the Earth-Moon system, but with eccentricity equal 0.1 , to see the effects of this parameter, and magnitude of the impulse $\delta V=$ $0.5 \mathrm{~km} / \mathrm{s}$. To understand the effects of the position of $M_{2}$ in the maneuvers, it is analyzed its efficiency at the true anomalies $v=0^{\circ}$ and $v=180^{\circ}$. In $v=0^{\circ}$ the secondary body is in its orbital periapsis around the primary. The velocity of $M_{2}$ is maximum due to the characteristics of an elliptical orbit, so it works in favor of increasing the magnitude of the energy variation. Considering the situation where $M_{2}$ is in the apoapsis of its orbit $\left(v=180^{\circ}\right)$, the velocity of $M_{2}$ is minimum, so the effects in the maneuver is reduced. Note that, for $v=0^{\circ}$ and $v=180^{\circ}, \beta=90^{\circ}$, therefore the region of loss of energy becomes to $0^{\circ}<\psi<180^{\circ}$ and the region of gains of energy is $180^{\circ}<\psi<360^{\circ}$, which is similar to the circular case.

Figure 9(a) shows the energy variation and Fig. 9(b) the efficiency of the powered Swing-By maneuver (in $\mathrm{km}^{2} / \mathrm{s}^{2}$ ) as a function of the periapsis radius of the orbit of the spacecraft (in $\mathrm{km}$ ), varying from approximately $1750 \mathrm{~km}$ to $2750 \mathrm{~km}$; and the approach angle, which defines the position of the periapsis of the incoming trajectory with respect to the secondary body. Figure 9(c) shows the same energy variation and Fig. 9(d) the efficiency of the powered SwingBy (in $\mathrm{km}^{2} / \mathrm{s}^{2}$ ), but now as a function of the periapsis radius of the orbit of the spacecraft (in $\mathrm{km}$ ) and the approach velocity of the spacecraft (in $\mathrm{km} / \mathrm{s}$ ).

Figure 9(a) quantifies the effects of the angle of approach and periapsis distance, showing nearly horizontal lines, which indicates the stronger importance of the angle of approach when compared to the periapsis distance. It can also be observed, from Fig. 9(b), that, for values of $\psi$ above approximately $250^{\circ}$, the powered Swing-By maneuver with the impulse applied in the periapsis is more efficient for the initial conditions used, when the goal is to increase the energy of the second orbit of the spacecraft after the close encounter. The magnitude of this efficiency increases with the angle of approach. It is important to note that the relevant particular case where $\psi=270^{\circ}$, the maximum gains of energy, is included in the regions of positive efficiency for the powered maneuver. This fact increases the applicability of the powered maneuver. Although very important, this particular case is not the only one that deserves to be considered. The angle of approach depends on the whole trajectory of the spacecraft around $M_{1}$, so it is not a free parameter. It means that, even if a large variation of energy is desired, it is not always possible to get $\psi=270^{\circ}$, due to other constraints in the trajectory of the spacecraft. Considering this point, it is important to map the regions of efficiency of the maneuver, even if the powered maneuver is recommended only for angles of approach not close to $\psi=270^{\circ}$.

There is also a dependency with the periapsis distance $r_{p}$, but this parameter has a smaller effect. This fact can be observed by the fact that most of the lines are nearly horizontals. The efficiency decreases with the periapsis distance, which is expected because the effect of the gravitational part of the maneuver decreases. With respect to the approach ve- 
Fig. 14 Analysis of the energy variation and efficiency in the energy gains region of the powered Swing-By for a system with $e=0.1, v=180^{\circ}, r_{p}=1.1$ radius of $M_{2}$ and $\delta V=0.5 \mathrm{~km} / \mathrm{s}$

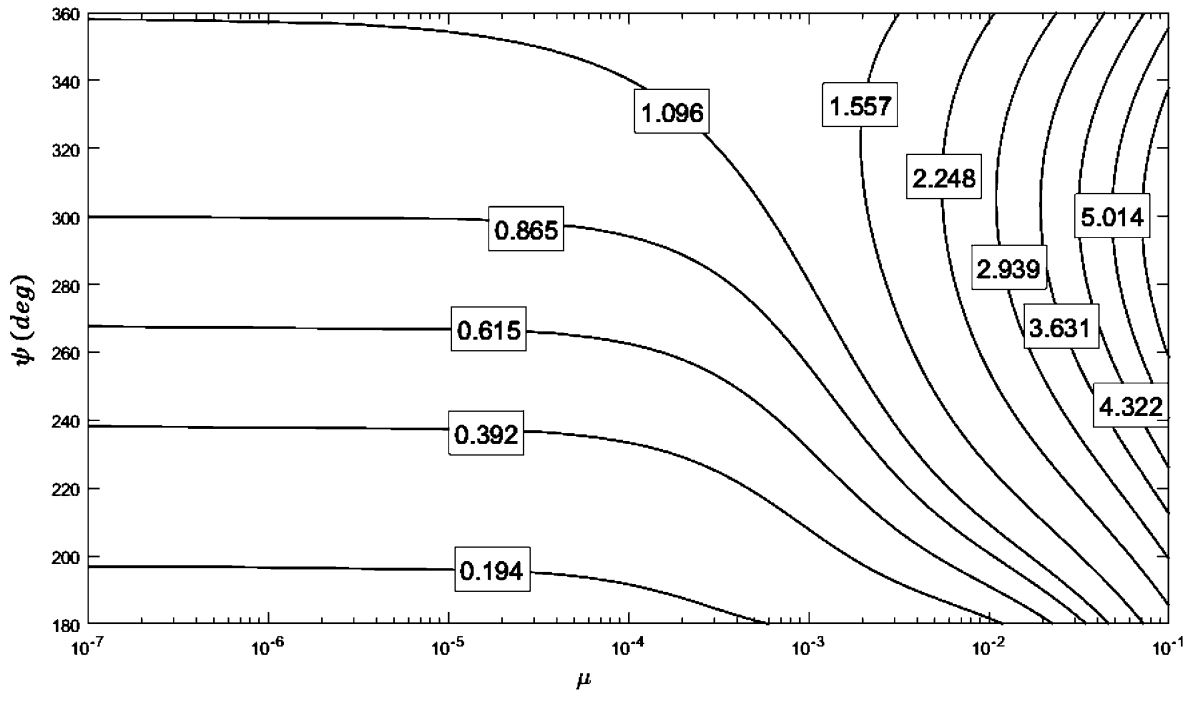

(a) $\Delta E_{\text {imp }}$ using $V_{\text {inf- }}=1.02 \mathrm{~km} / \mathrm{s}$

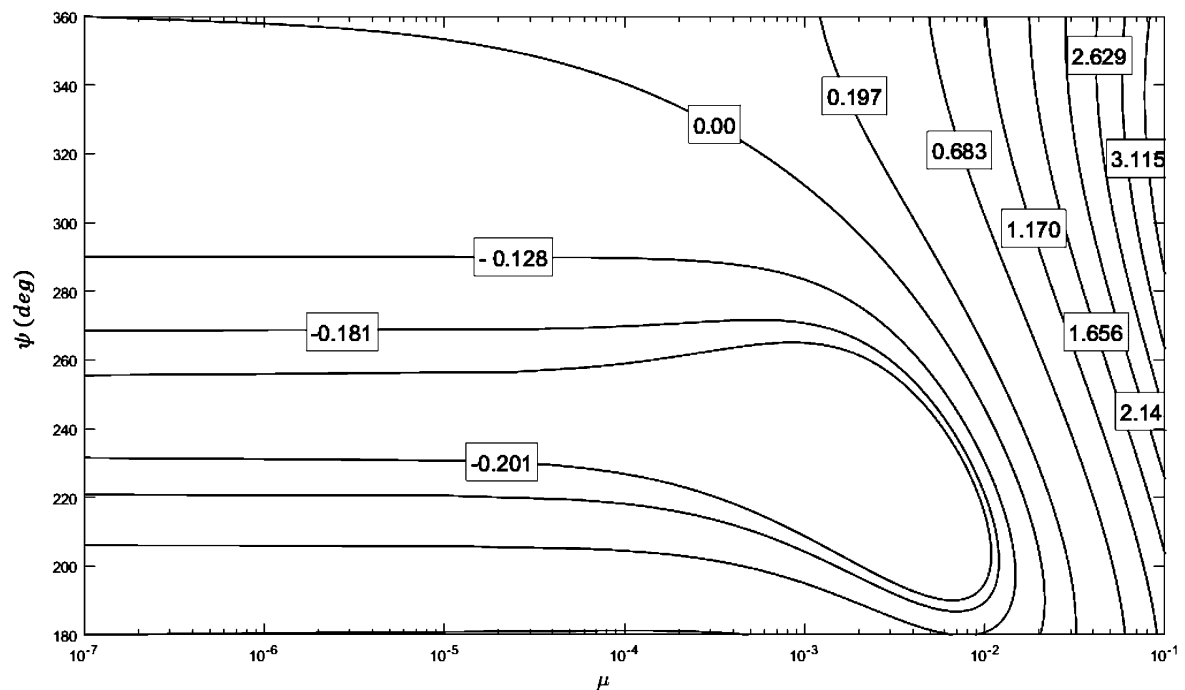

(b) Efficiency using $V_{\text {inf- }}=1.02 \mathrm{~km} / \mathrm{s}$

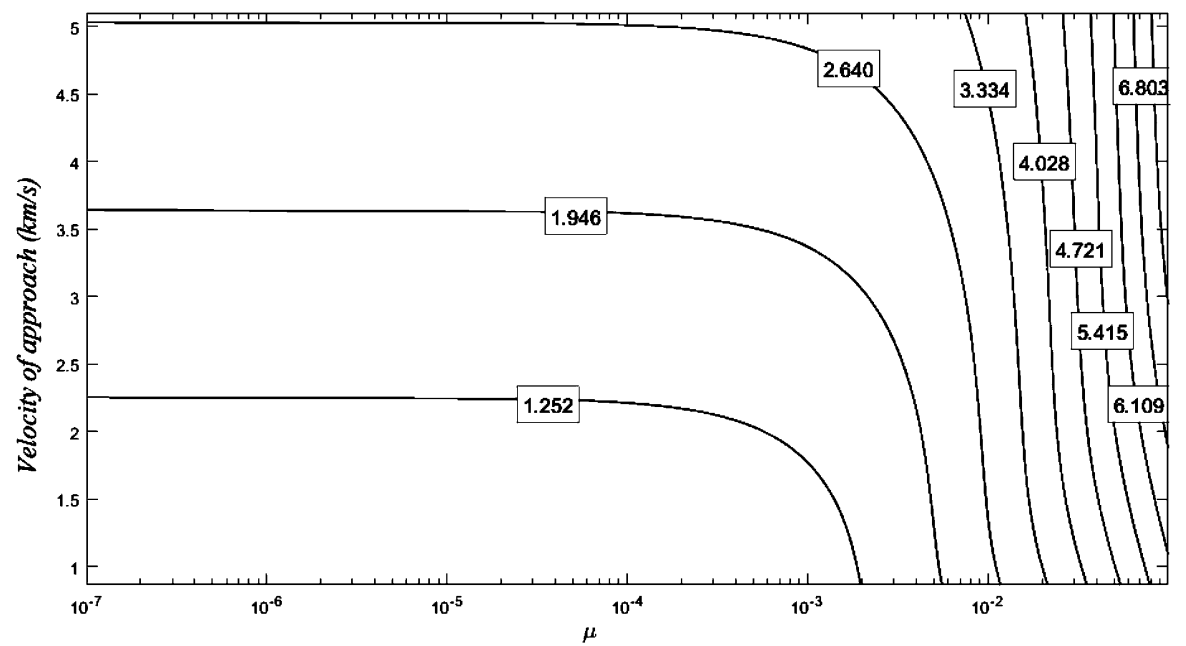

(c) $\Delta E_{\text {imp }}$ using $\psi=270^{\circ}$ 
Fig. 14 (Continued)

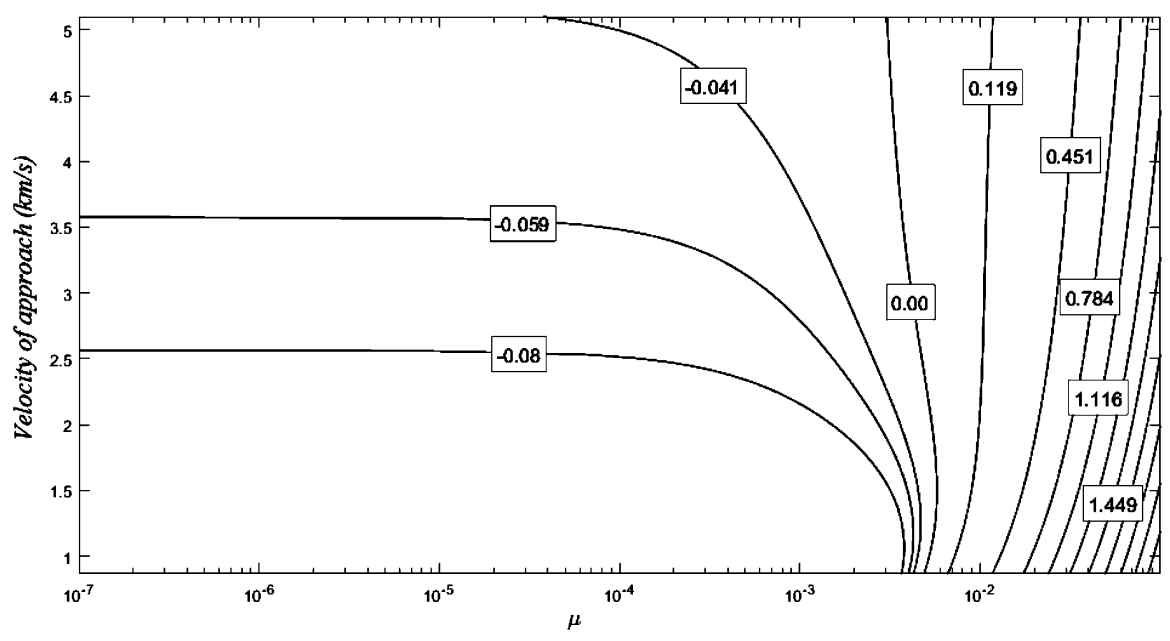

(d) Efficiency using $\psi=270^{\circ}$

locity, Fig. 9(d) shows that the powered Swing-By is more efficient, considering the case $\psi=270^{\circ}$, for all values of

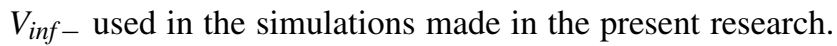
Smaller values of the periapsis radius result in higher magnitudes of the efficiency. It is also observed the existence of the regions: (i) the one where the velocity of approach is below $2 \mathrm{~km} / \mathrm{s}$, where the lines are almost horizontals and the velocity of approach dominates the maneuver, with small effects coming from the periapsis distance; and (ii) the region where the velocity of approach is above $2 \mathrm{~km} / \mathrm{s}$, where the lines are nearly verticals, so the velocity of approach has small effects in the maneuvers, with the periapsis distance dominating the results. Figure 9(c) quantifies the effects of the velocity of approach and the periapsis distance, with the inclined lines indicating that both parameters have similar effects in the maneuver. The variations of energy increase with the velocity of approach and decreases with the periapsis distance.

Figure 10 is made using the same conditions used in Fig. 9, but now $M_{2}$ is in the apoapsis of the orbit $\left(v=180^{\circ}\right)$. Note that the behaviors of the results are similar for the case with $v=0^{\circ}$. The main difference is in the magnitude of the energy variation, which are smaller due to the reduction in $V_{2}$.

Figures 11 and 12 present the results for a maneuver where the goal is to remove the energy from the spacecraft. In Fig. 11(a), for $v=0^{\circ}$, and Fig. 11(c), for $v=180^{\circ}$, the energy variations of the powered maneuver are presented. Since the objective is to remove energy from the spacecraft, the initial conditions used a geometry that leads to energy losses. In this way, there are negative variations in the energy, and the spacecraft decreased the energy of the second orbit, after the impulse is applied, when compared to the first orbit. This case also has similar results, with the angle of approach dominating the maneuver with respect to the periapsis distance (see Fig. 11(a)) and with equilibrium in the effects of the velocity of approach and the periapsis distance (see Fig. 11(b)). Figures 11(b) and 11(d) show the efficiency of the powered maneuver. Note that the best cases occur for $\psi$ up to approximately $95^{\circ}$, with the highest efficiency at $\psi$ close to zero. This is because at $\psi=0^{\circ}$ the effect of gravity on the Swing-By maneuver is null, so $\Delta E_{a f t e r}$ is smaller. Figure 11(b) shows that the angle of approach is more important in defining the efficiency of the maneuvers, compared to the periapsis distance, which is observed by the fact that the lines representing the energy variations are linearly horizontals. Figure 11(d) shows two regions: (i) one with velocity of approach below $2 \mathrm{~km} / \mathrm{s}$, where the lines are horizontals and the velocity of approach dominates the variations of energy, leaving small effects for the periapsis distance, and (ii) a different region, with velocity of approach above $2 \mathrm{~km} / \mathrm{s}$, where the lines are inclined and the velocity of approach and periapsis distances have equal effects in the variations of energy.

Next, Fig. 12 shows the best conditions when the goal is to remove energy from the spacecraft. The efficient maneuvers occur when $\psi$ is less than $100^{\circ}$ and for all the values of $V_{\text {inf }}$ - greater than approximately $1.6 \mathrm{~km} / \mathrm{s}$ (Fig. 12(d)). Velocities of approach smaller than $1.6 \mathrm{~km} / \mathrm{s}$, for the conditions used, result in captures or collisions of the spacecraft with the secondary body. For these conditions there is also a dependency with the periapsis distance $r_{p}$. The behaviors are very similar to the previous cases, just with differences in the magnitude of the energy variations, so there is no need for detailed explanations.

To maximize the effect of the maneuver, whether for the gain or loss of energy, we consider the secondary body in the periapsis of its orbit around the primary. If the apoapsis is used, for $e=0.1$, there is a difference of up to approximately $0.35 \mathrm{~km}^{2} / \mathrm{s}^{2}$ in the energy gains and up to approximately $0.3 \mathrm{~km}^{2} / \mathrm{s}^{2}$ in the energy losses situation. Once the maneuver is made with $M_{2}$ at the periapsis, we know 
Fig. 15 Analysis of the energy variation and efficiency in the energy losses region of the powered Swing-By for a generic system with $e=0.1, v=0^{\circ}$, $r_{p}=1.1$ radius of $M_{2}$ and $\delta V=0.5 \mathrm{~km} / \mathrm{s}$

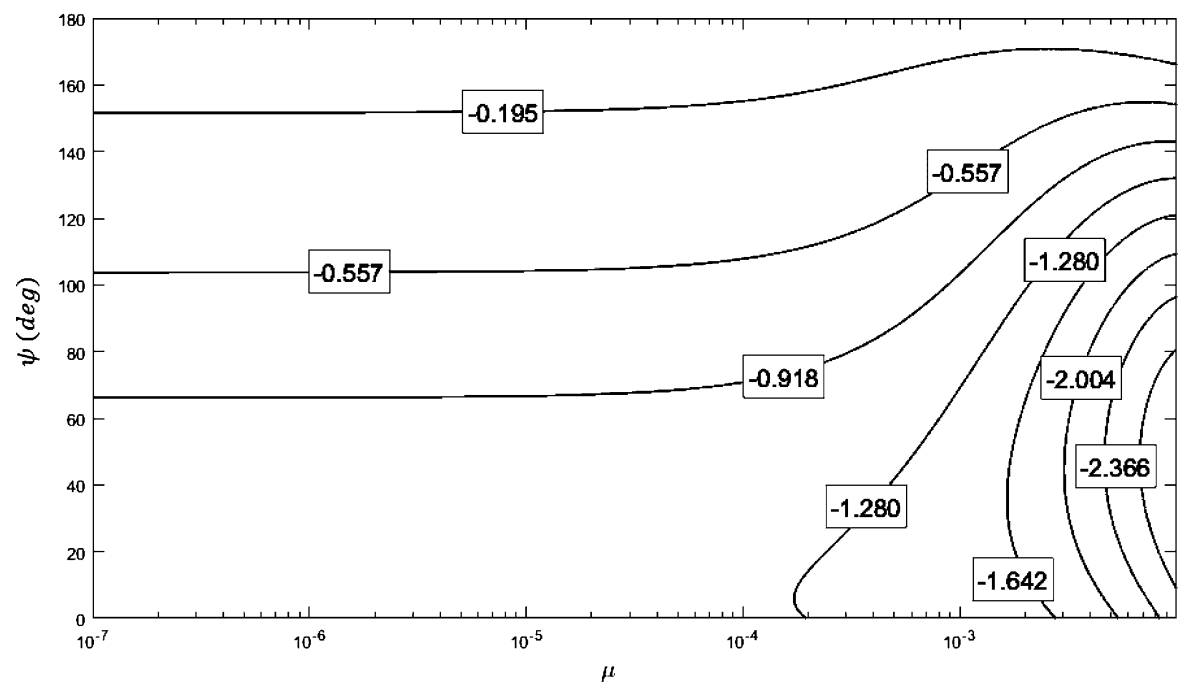

(a) $\Delta E_{\text {imp }}$ using $V_{\text {inf- }}=1.6 \mathrm{~km} / \mathrm{s}$

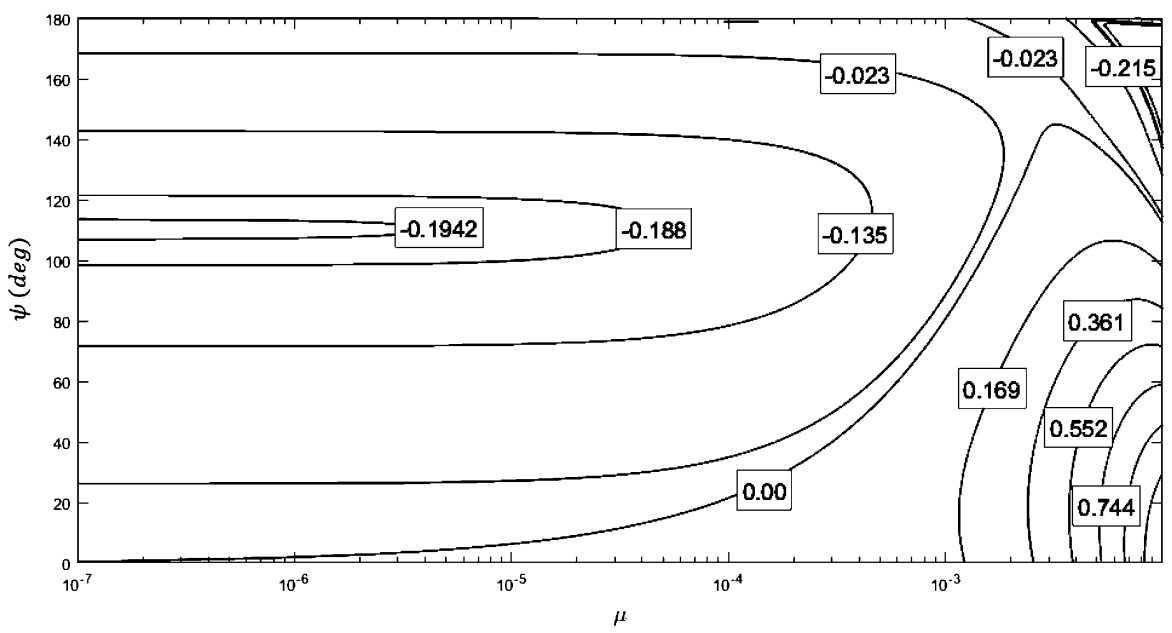

(b) Efficiency using $V_{\text {inf- }}=1.6 \mathrm{~km} / \mathrm{s}$

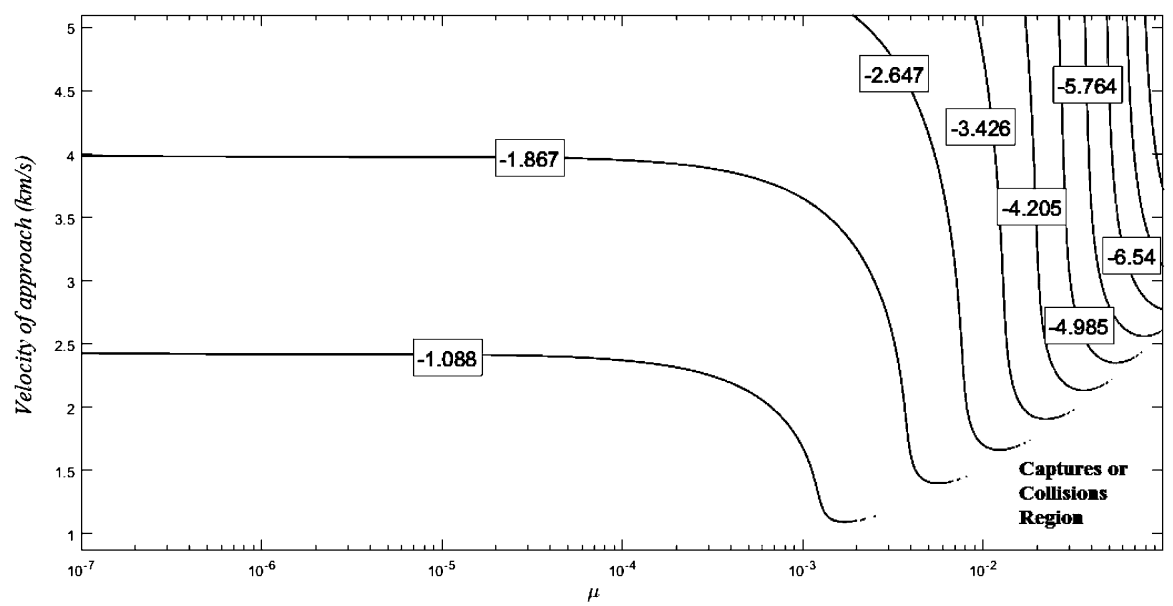

(c) $\Delta E_{\text {imp }}$ using $\psi=90^{\circ}$ that the region of efficiency for the energy gains occurs at approximately $\psi>250^{\circ}$ for $V_{\text {inf }-}=1.02 \mathrm{~km} / \mathrm{s}$ and for all values of $V_{i n f}-$ when $\psi=270^{\circ}$, for all $r_{p}$ studied. For the energy loss situation, the efficient region is at approxi- mately $\psi<100^{\circ}$, for $V_{i n f-}=1.6 \mathrm{~km} / \mathrm{s}$, and $V_{i n f-}$ from 1.6 to $5.0 \mathrm{~km} / \mathrm{s}$ for $\psi=90^{\circ}$, for all values of $r_{p}$. If the maneuver is made with $v=180^{\circ}$, with the goal of increasing the energy gains, $\psi$ must be larger than $230^{\circ}$ for all values 
Fig. 15 (Continued)

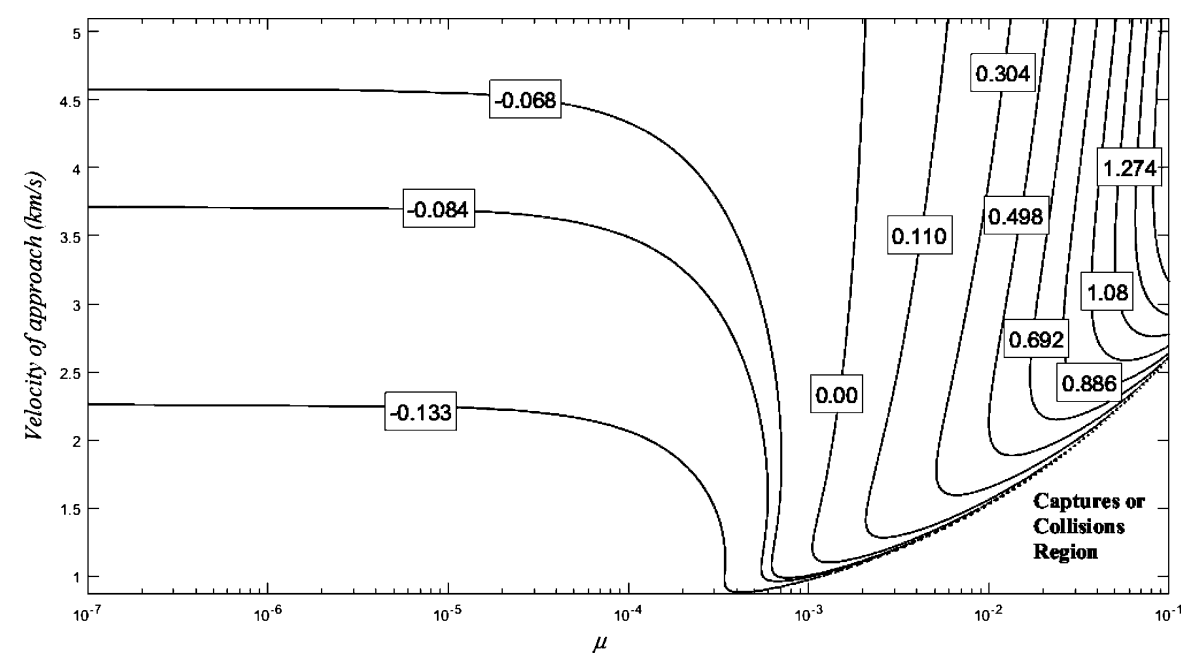

(d) Efficiency using $\psi=90^{\circ}$

of $r_{p}$ and $V_{\text {inf- }}=1.02 \mathrm{~km} / \mathrm{s}$; and $V_{\text {inf- }}$ needs to go from 1.6 to $5.0 \mathrm{~km} / \mathrm{s}$ for $\psi=270^{\circ}$. For the energy loss situation, $\psi<100^{\circ}$ for $V_{\text {inf- }}=1.6 \mathrm{~km} / \mathrm{s}$, and $V_{\text {inf }}$ is in the interval from 1.6 to $5.0 \mathrm{~km} / \mathrm{s}$ for $\psi=90^{\circ}$, for all $r_{p}$ studied. In these regions it is recommended to apply the impulse when the spacecraft is passing by the periapsis of its orbit around the secondary body.

Next, a study is made to measure the efficiency of the powered Swing-By maneuver as a function of the gravitational parameter of the secondary body, the approach angle (degrees) and the velocity of approach $(\mathrm{km} / \mathrm{s})$. This is important to verify which systems of main bodies can benefit from the powered maneuver. The energy variations for the powered maneuver are also presented. Figure 13(a) and 13(c) show the energy variation of the powered maneuver. They have two distinct regions: (i) one where the mass parameter is above $10^{-2}$, where the lines are nearly verticals, indicating that the mass parameter gives stronger effects in the energy variations, compared to the angle of approach, and (ii) a different region, where the mass parameter is below $10^{-2}$, where the lines are nearly horizontals, indicating that the mass parameter give almost zero effects in the energy variations, making the angle of approach the dominating variable. Figure 13(b) and 13(d) show that the efficiency of the powered maneuver is highly dependent on the gravitational parameter of the secondary body. Note that the efficiency is positive in the top-right region of the plot (Fig. 13(b)), which means higher values of the mass parameter and the angle of approach. For values of $\mu$ approximately greater than $10^{-2}$ the powered maneuver is advantageous for most of the values of the angle of approach. For $\psi$ above $270^{\circ}$ this region increases, including some values of $\mu$ less than $10^{-2}$, which goes to values much smaller for higher values of the angle of approach. Figure 13(d) shows the efficiency as a function of $\mu$ and $V_{\text {inf-. }}$. The limit between an efficient maneuver is
$E f=0$ and it occurs near $\mu=5 \times 10^{-3}$ for all values of $V_{\text {inf- }}$. The same two regions divided by the mass parameter $10^{-2}$ occurs, shifting between horizontal and vertical lines, as explained above.

The physical explanations for those observations are related to the effects of the close approach. Celestial bodies with larger masses can provide larger variations of energy, so the powered maneuver can help the spacecraft to get more energy of the gravity part of the maneuver, so generating better efficiency for the powered maneuver. On the other side, small bodies do not give much energy to the spacecraft and the impulse applied after the maneuver can give larger levels of energy. This fact combined with the already explained condition that the angle of approach is not a free parameter in the mission, makes of the present study a very important guide to inform under which circumstances it is better to use the powered maneuver.

Next, Fig. 14 shows the energy variation and efficiency of the powered maneuver for secondary body in the apoapsis of its orbit in the close approach moment. The results have the same behavior, just showing a reduction of the energy variations due to the smaller value of $V_{2}$.

To complete this general study, Figs. 15 and 16 present the energy variations and efficiency of the powered maneuver realized when the secondary body is in the periapsis and the apoapsis of its orbit, respectively, when the goal is to remove energy of the spacecraft. The general results are very similar, so it is not necessary to repeat explanations. The main difference is the appearance of larger regions of captures and collisions generated by the reduction of energy of the spacecraft. Figure 15(b) shows that the best conditions are obtained for $\mu$ greater than approximately $10^{-3}$ and approach angle less than around $140^{\circ}$. For the conditions used, the region of $\mu>10^{-2}$ resulted in captures or collisions of the spacecraft by the $M_{2}$. Figure 15(d) shows that applying 
Fig. 16 Analysis of the energy variation and efficiency in the energy losses region of the powered Swing-By for a generic system with $e=0.1, v=180^{\circ}$, $r_{p}=1.1$ radius of $M_{2}$ and $\delta V=0.5 \mathrm{~km} / \mathrm{s}$

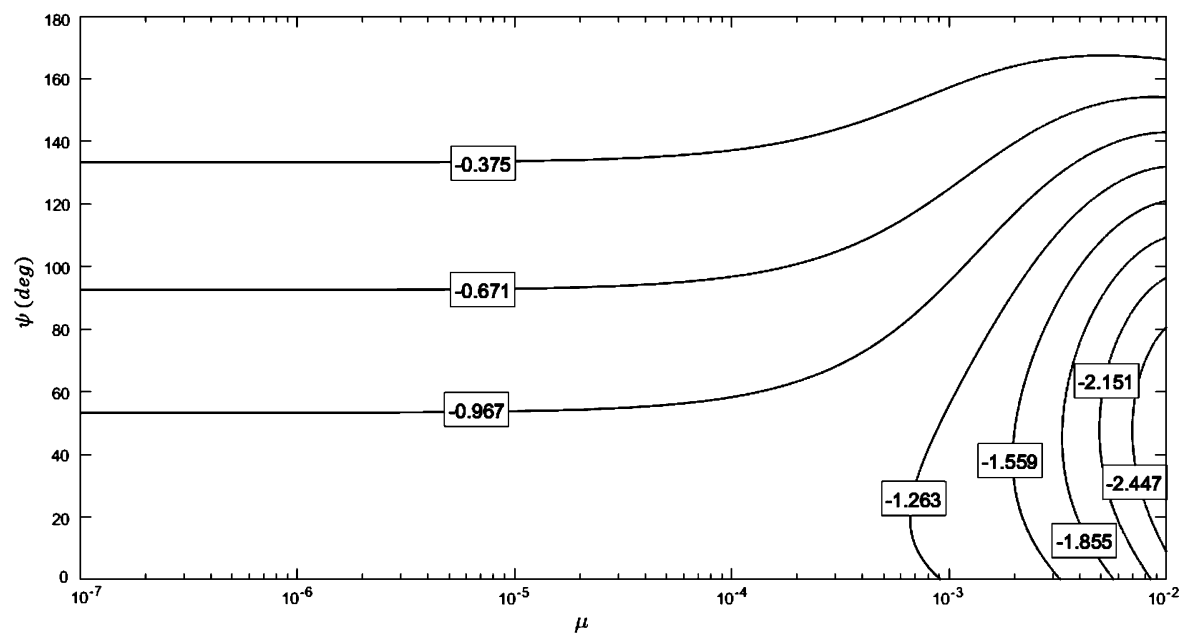

(a) $\Delta E_{\text {imp }}$ using $V_{\text {inf- }}=1.6 \mathrm{~km} / \mathrm{s}$

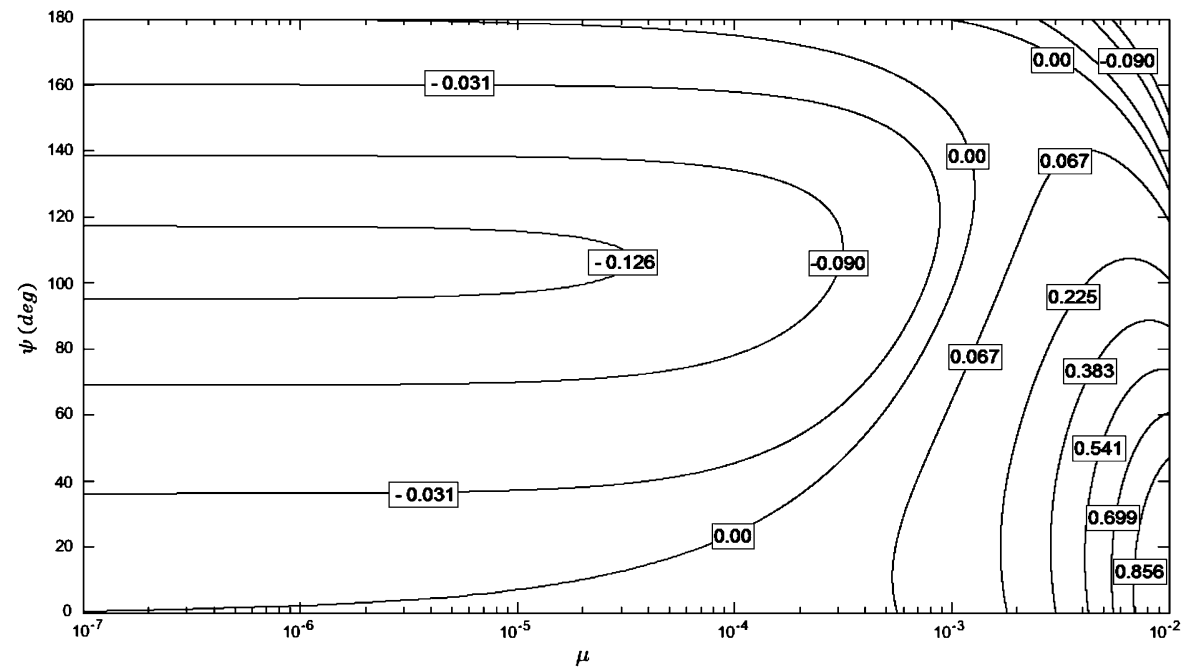

(b) Efficiency using $V_{\text {inf- }}=1.6 \mathrm{~km} / \mathrm{s}$

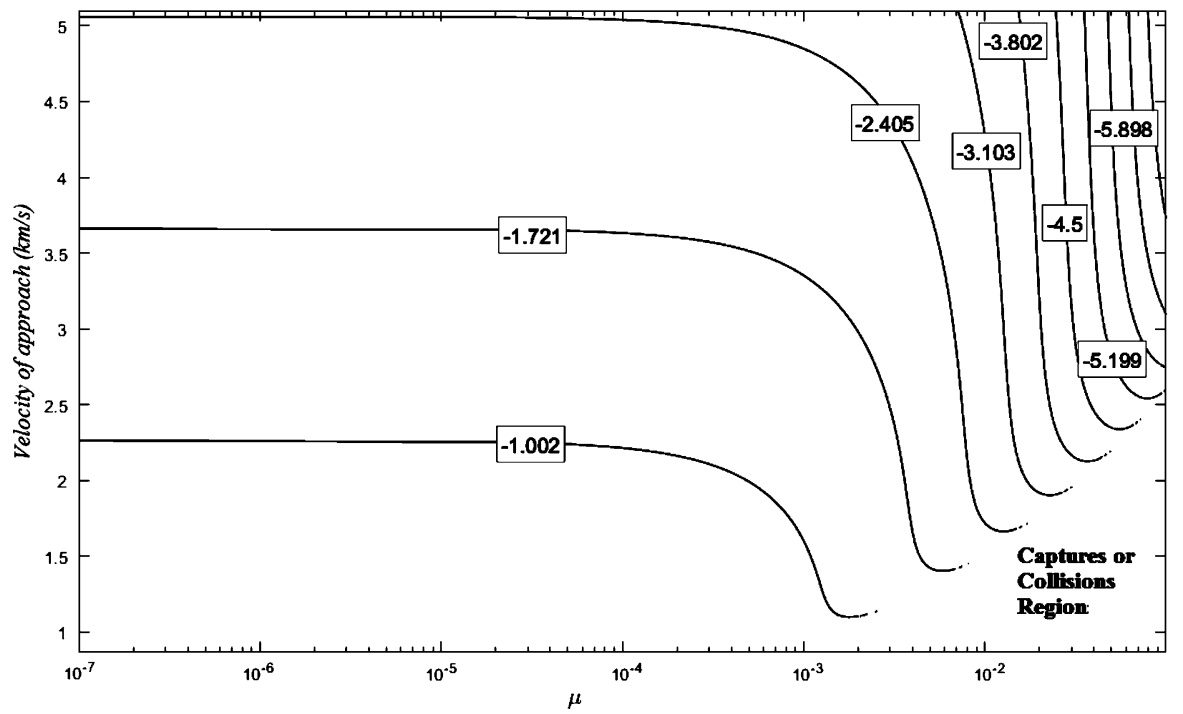

(c) $\Delta E_{\text {imp }}$ using $\psi=90^{\circ}$ 
Fig. 16 (Continued)

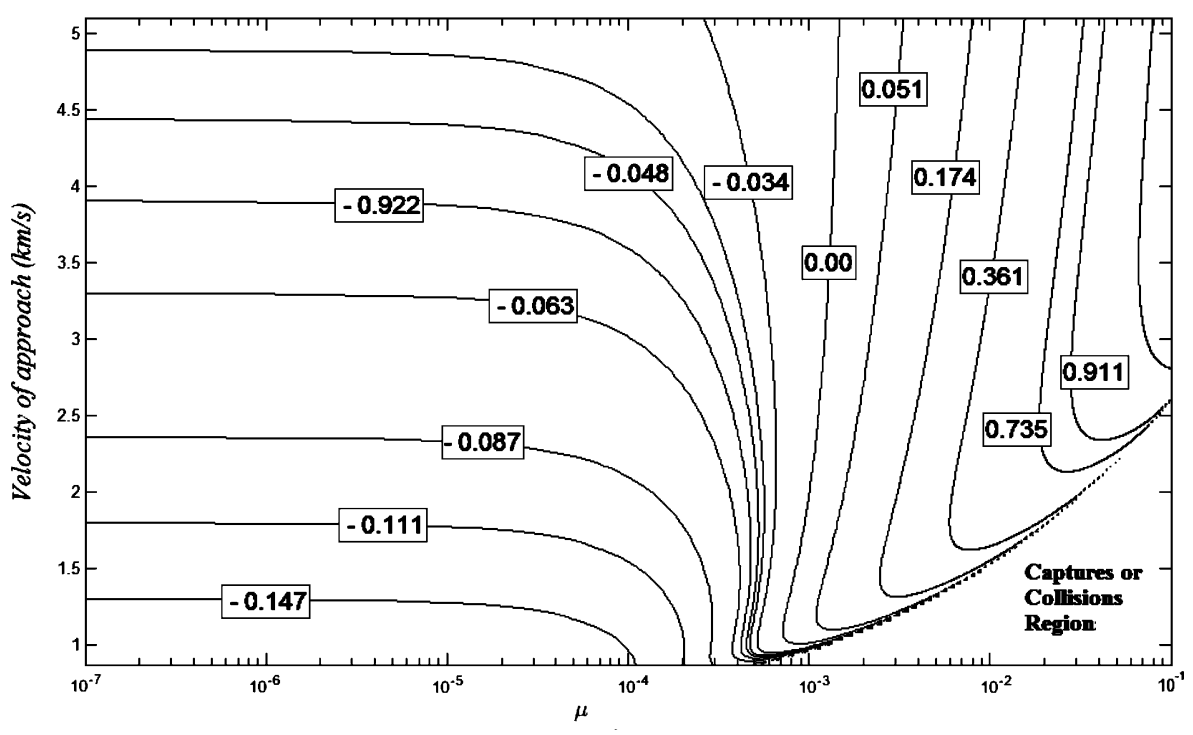

(d) Efficiency using $\psi=90^{\circ}$

the impulse in the periapsis of the orbit can be more efficient for values of $\mu$ greater than $10^{-3}$, but for approach velocities up to approximately $2.5 \mathrm{~km} / \mathrm{s}$ the energy loss can result in captures or collisions.

Figure 16(b) shows that the best conditions are obtained for $\mu$ approximately greater than $10^{-3}$. For some values of the approach angle (greater than approximately $160^{\circ}$ and less than $30^{\circ}$ ) the positive region includes smaller values of the gravitational parameter. For the conditions used, the region of $\mu>10^{-2}$ resulted in captures or collisions of the spacecraft by $M_{2}$. Figure 16(d) shows that applying the impulse in the periapsis of the orbit can be more efficient for values of $\mu$ greater than $10^{-3}$, when the objective is to decrease the spacecraft energy, but for some values of approach velocity this energy loss can result in captures or collisions.

\section{Real systems}

The next step of the present research is to show results for some real systems. To do that, some examples for the SunMercury and Sun-Mars systems are presented. For both systems, the velocity of approach ( $\left.V_{\text {inf- }}\right)$ used is $25 \%$ above the minimum possible value for a spacecraft to reach the secondary body coming from the Earth in a Hohmann type maneuver and the gravitational parameter is of the order of $10^{-7}$. Mercury has an eccentricity equal 0.2056 and Mars equal 0.0934 . The impulse adopted is $\delta V=0.5 \mathrm{~km} / \mathrm{s}$. Figure 17 shows the energy variation of the powered Swing-By and the efficiency of this maneuver for the Sun-Mercury system with $v=0^{\circ}$ and $\delta V=0.5 \mathrm{~km} / \mathrm{s}$ in the situation where the goal is to gain energy.
The results are aligned with the general studies made up to know in the present paper. Note also that the best results that have positive efficiency are for approach angle above approximately $340^{\circ}$. For $\psi=350^{\circ}$, to apply the impulse in the periapsis with approach velocity approximately above $20 \mathrm{~km} / \mathrm{s}$ is not recommended. Figure 18 presents the analysis for the loss of energy. In this case the best region in $\psi$ is approximately from $0^{\circ}$ to $30^{\circ}$.

Figure 19 shows the energy variation of the powered Swing-By and efficiency for Sun-Mars system with $v=0^{\circ}$ and $\delta V=0.5 \mathrm{~km} / \mathrm{s}$ when the goal is energy gain. The results are similar to the previous ones.

To apply the impulse in the periapsis of the orbit with $\psi$ above $300^{\circ}$ is more recommended in this case. There is a large region with positive efficiency in terms of $V_{\text {inf }}$. Note also the positive regions for $V_{\text {inf }}$ - up to approximately $18 \mathrm{~km} / \mathrm{s}$, for $\psi=340^{\circ}$. The results for the energy loss in the Sun-Mars system are shown in Fig. 20. For the energy loss situation, the most recommended is $\psi$ from 0 to about $50^{\circ}$.

In these cases, to apply the impulse in the periapsis of the spacecraft orbit with $M_{2}$ is more efficient for $\psi$ different from $90^{\circ}$ or $270^{\circ}$, the optimum regions. As already explained, although very important, the particular cases of the Swing-By maneuver for $\psi=270^{\circ}$, when the goal is to gain energy, and $\psi=90^{\circ}$, when the goal is to lose energy, are not the only ones that should be considered. The approach angle is not a free parameter in a mission. It depends on the whole spacecraft trajectory around $M_{1}$, so maneuvers with $\psi=90^{\circ}$ or $\psi=270^{\circ}$ are not always possible. This fact justifies the study of maneuvers even in systems where they are not efficient in those regions. 


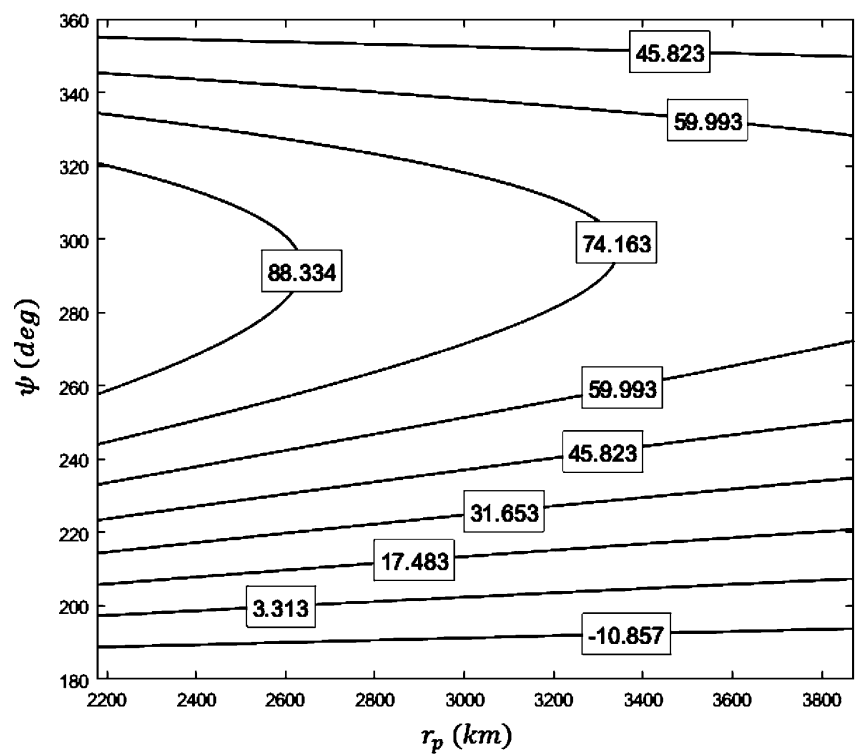

(a) $\Delta E_{\text {imp }}$ using $V_{\text {inf- }}=12.1 \mathrm{~km} / \mathrm{s}$

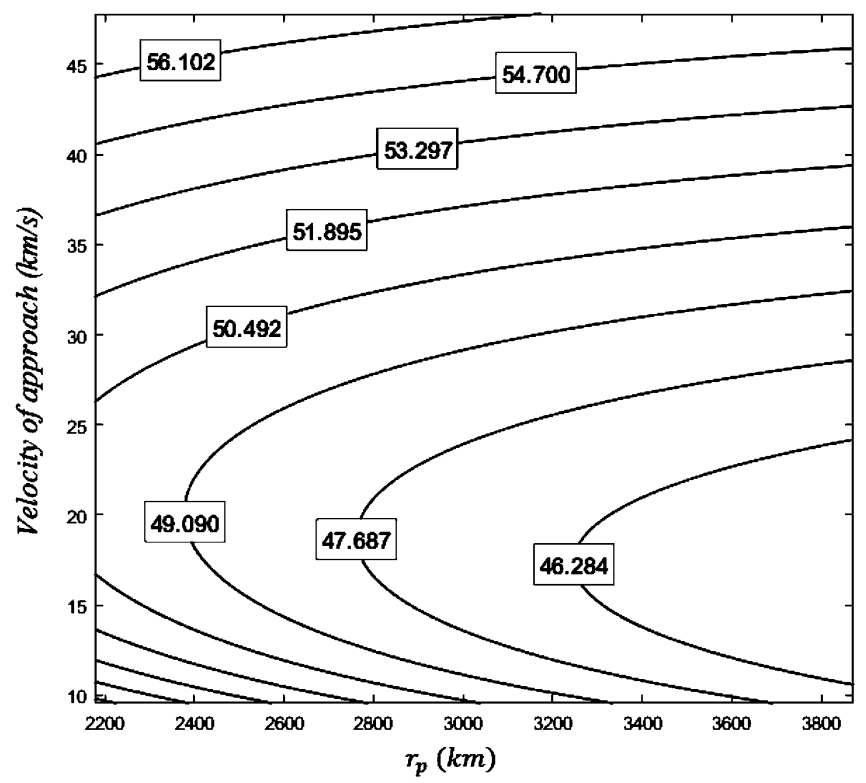

(c) $\Delta E_{\text {imp }}$ using $\psi=350^{\circ}$

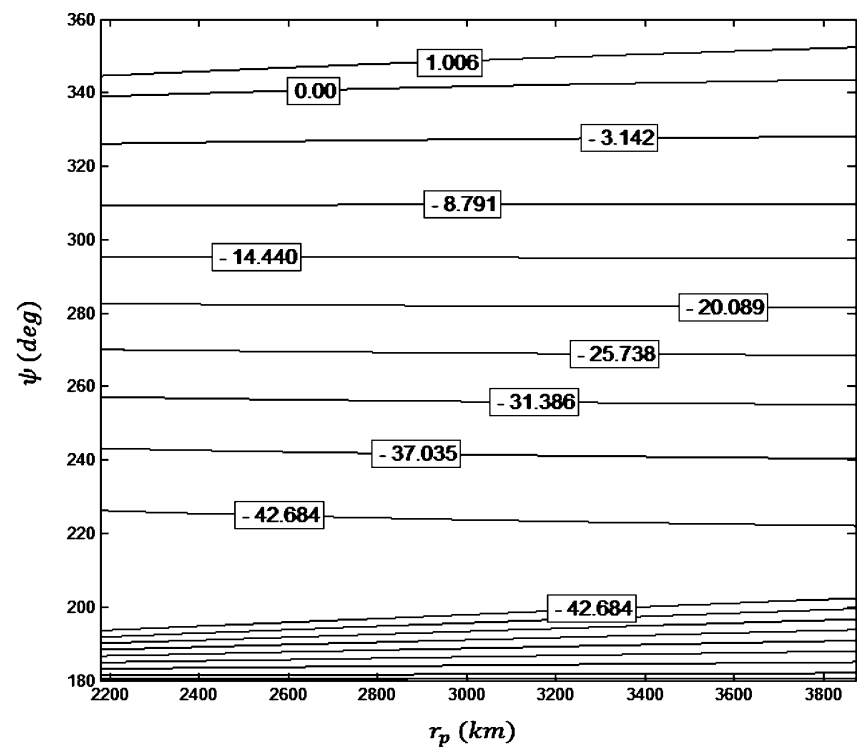

(b) Efficiency using $V_{\text {inf }-}=12.1 \mathrm{~km} / \mathrm{s}$

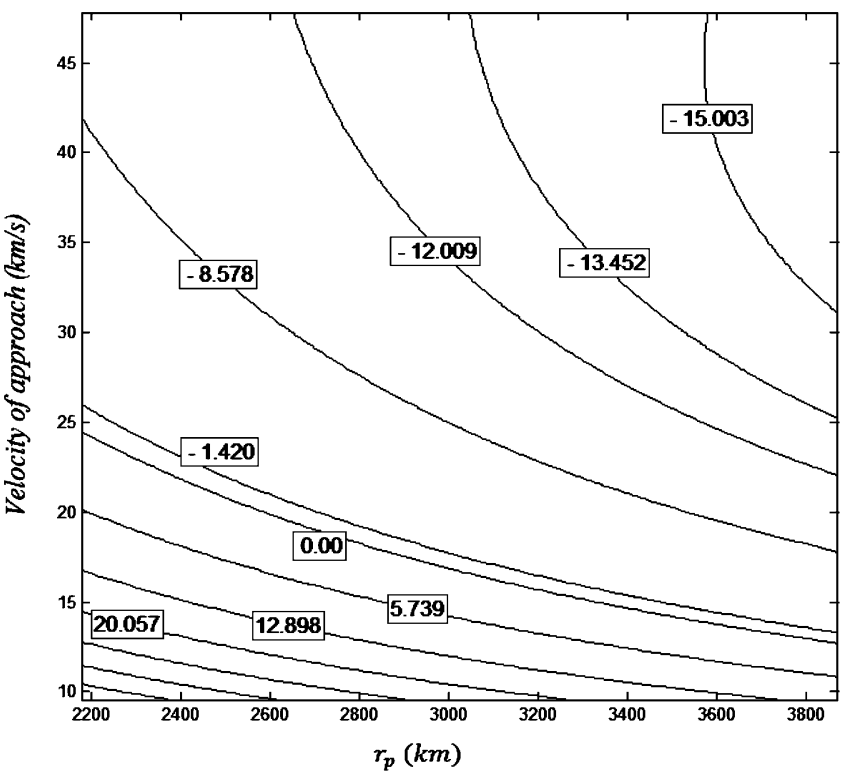

(d) Efficiency using $\psi=350^{\circ}$

Fig. 17 Analysis of the energy variation and efficiency in the energy gains region of the powered Swing-By for the Sun-Mercury system with $v=0^{\circ}$ and $\delta V=0.5 \mathrm{~km} / \mathrm{s}$

\section{Conclusions}

The limitations and real gains of the Swing-By maneuver with the impulse applied in the orbital periapsis of the spacecraft were presented. From some general observations about the efficiency of the maneuver, it is possible to say that the gravitational parameter of the secondary body has an important influence on the results, as well as the position of the secondary body in the elliptical orbit, the approach angle and the approach velocity. Efficiency has a smaller dependence with the radius of the periapsis. There are cases where the impulsive maneuver is more efficient than the pure maneuver with the impulse applied outside the sphere of influence of $M_{2}$ and other situations where it is not. The results were presented for different initial conditions, mapping the best conditions for a generic system.

The modification made in the trajectory of a spacecraft by the gravity of the body and the impulse applied in the powered Swing-By maneuver was analyzed as a function of the velocity and energy variation in this work. This information was presented with respect to the main body of the system. From the equations obtained, it is only possible to 


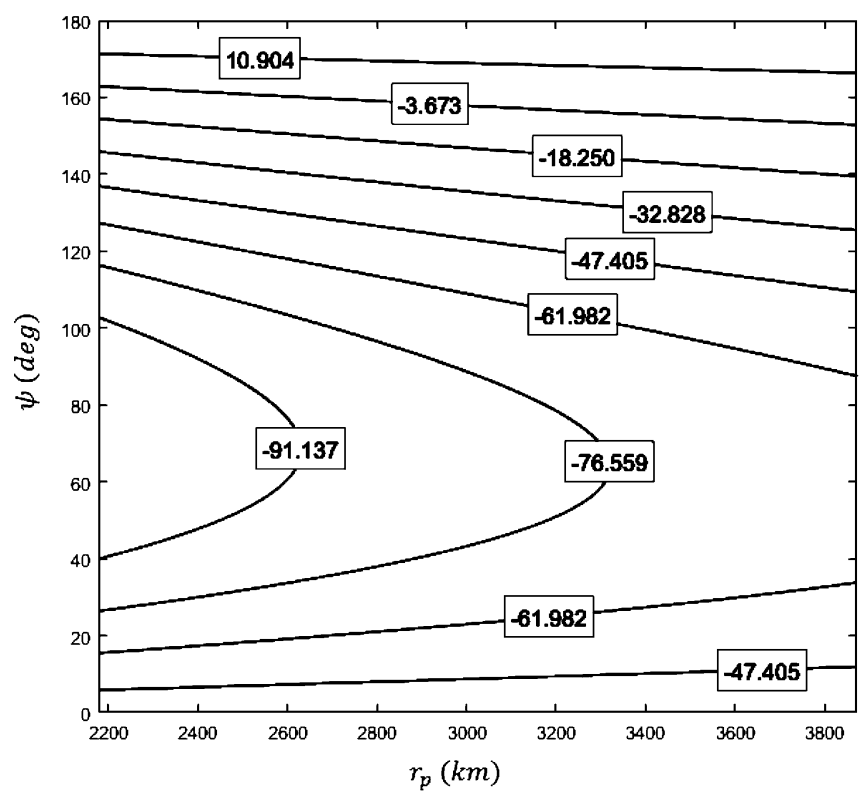

(a) $\Delta E_{\text {imp }}$ using $V_{\text {inf- }}=12.1 \mathrm{~km} / \mathrm{s}$

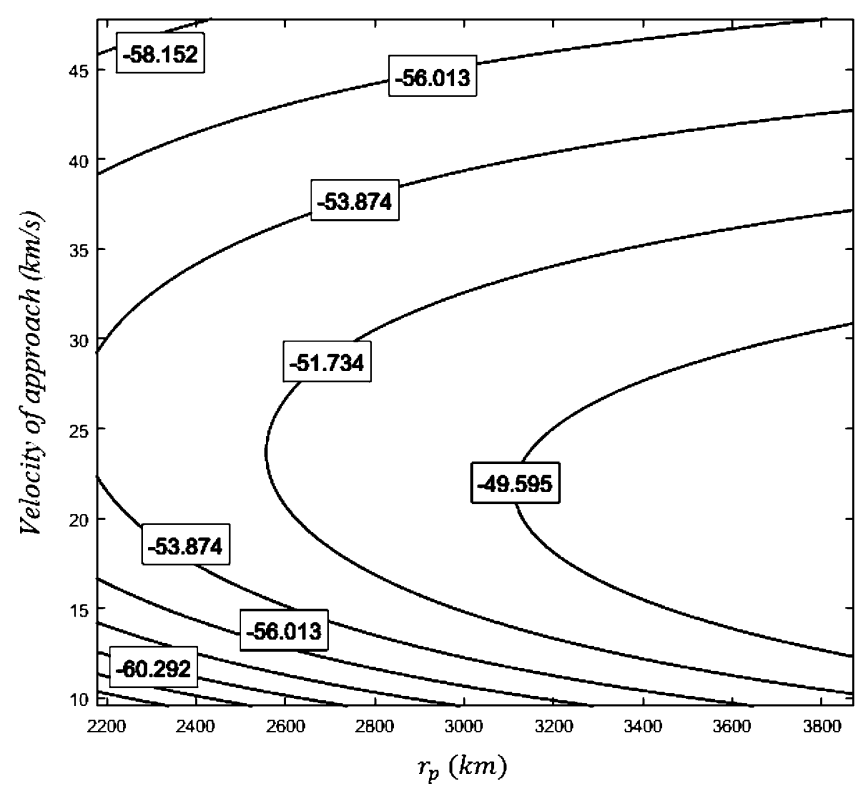

(c) $\Delta E_{\text {imp }}$ using $\psi=15^{\circ}$

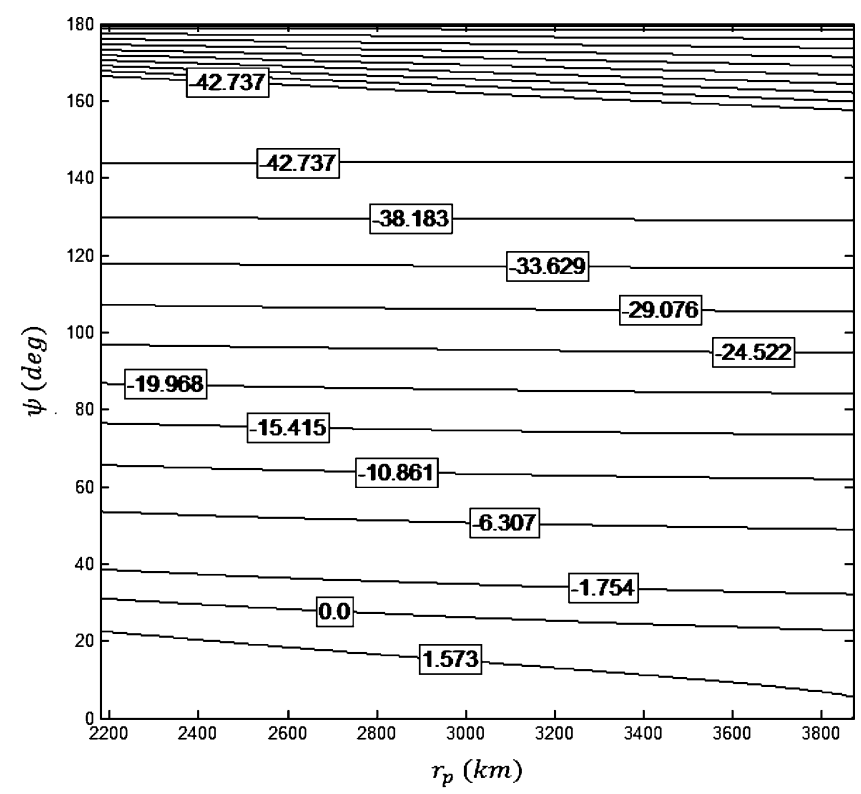

(b) Efficiency using $V_{\text {inf- }}=12.1 \mathrm{~km} / \mathrm{s}$

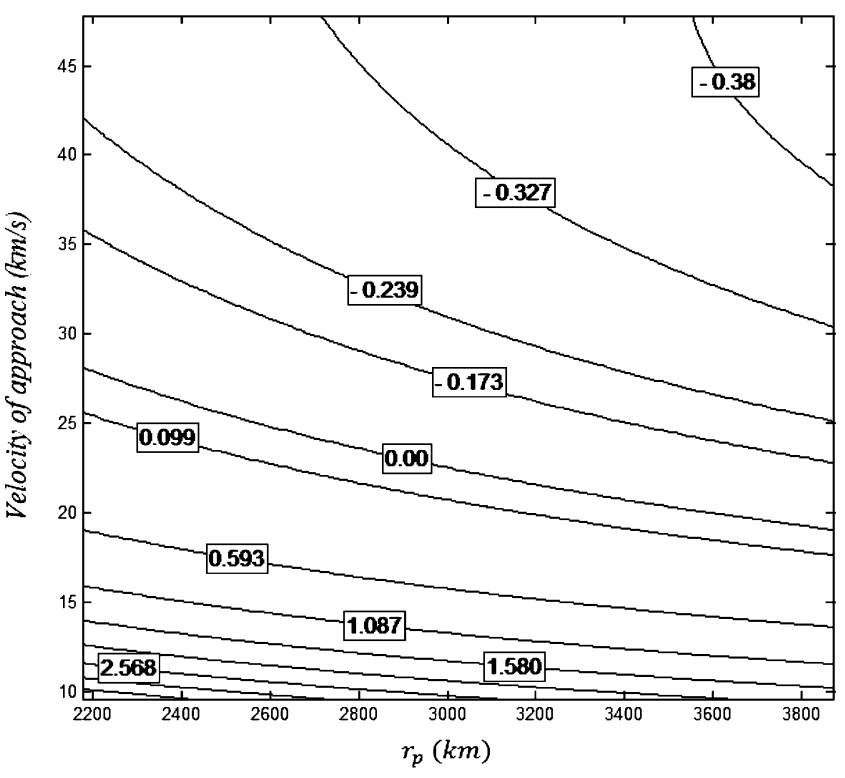

(d) Efficiency using $\psi=15^{\circ}$

Fig. 18 Analysis of the energy variation and efficiency in the energy losses region of the powered Swing-By for the Sun-Mercury system with $\nu=0^{\circ}$ and $\delta V=0.5 \mathrm{~km} / \mathrm{s}$

identify trajectories that did not escape from the orbit around the secondary body, so it is not possible to discriminate between capture and collision.

The application of an impulsive maneuver combined with a close approach is recommended in situations where only the gravity of the body is not enough to achieve the objective of the mission. This type of maneuver focuses on the minimum expenditure of fuel by choosing the best moment to apply the impulsive maneuver. The possibility of variation in the magnitude and direction of the impulse also helps to maximize its effect.
The solutions obtained showed that, if the objective is to maximize the energy gain, to apply the impulse in the direction non-tangential to the orbit can give better results. For the initial conditions studied, to apply the impulse towards the secondary body makes the spacecraft to move closer to the body, working in favor of increase the gains coming from the gravity part of the maneuver.

With respect to the eccentricity of the orbit of the main bodies, it is well known that the energy variation is directly dependent on this parameter. The velocity variation does not 


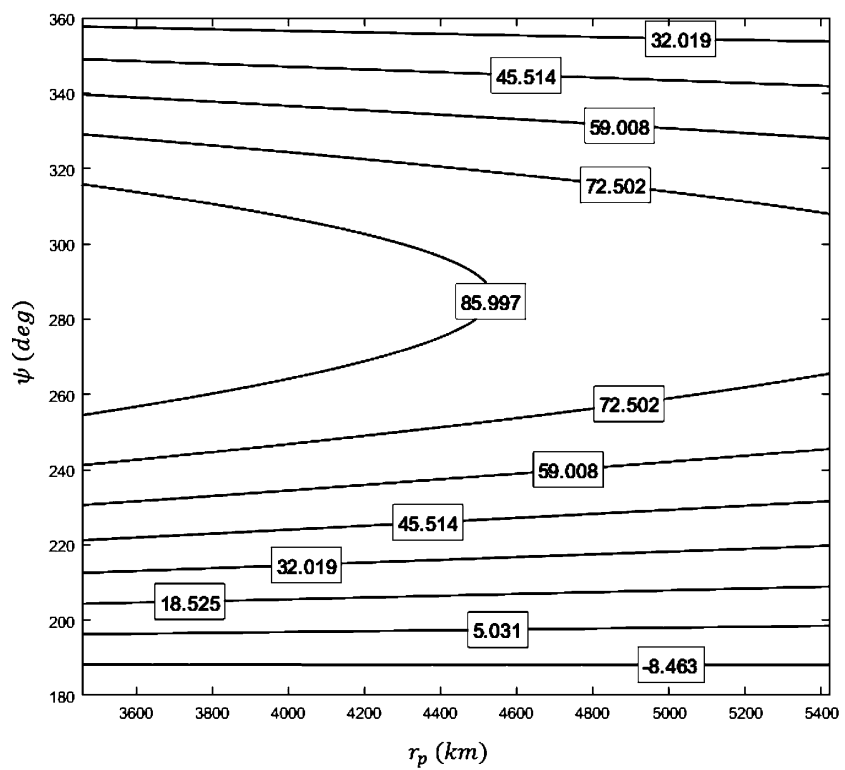

(a) $\Delta E_{\text {imp }}$ using $V_{\text {inf- }}=3.4 \mathrm{~km} / \mathrm{s}$

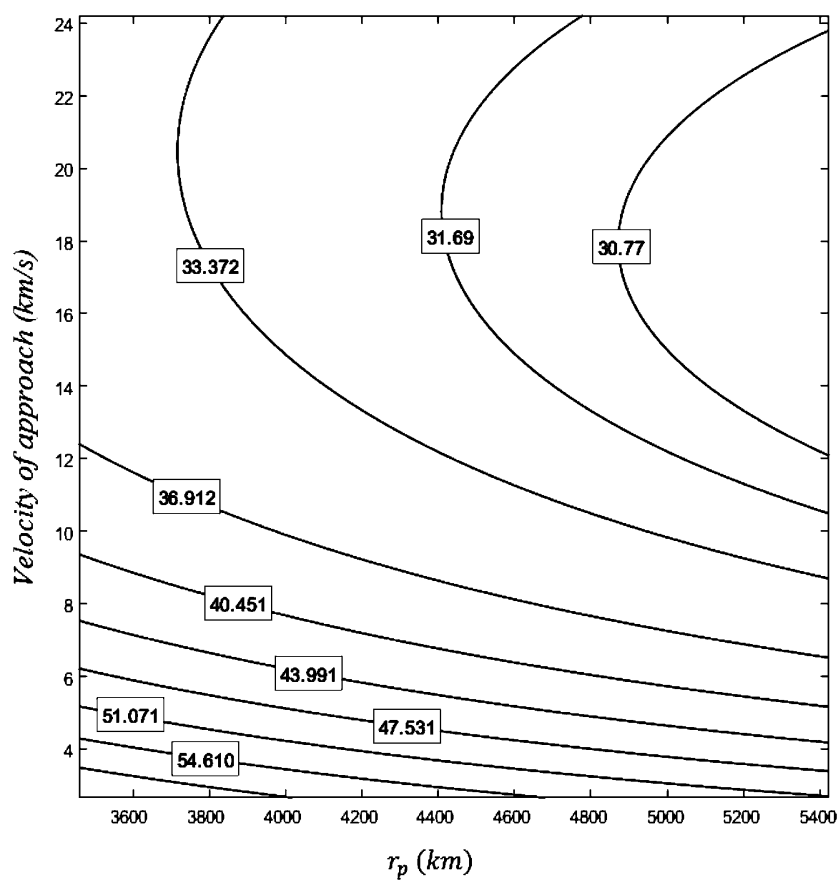

(c) $\Delta E_{\text {imp }}$ using $\psi=340^{\circ}$

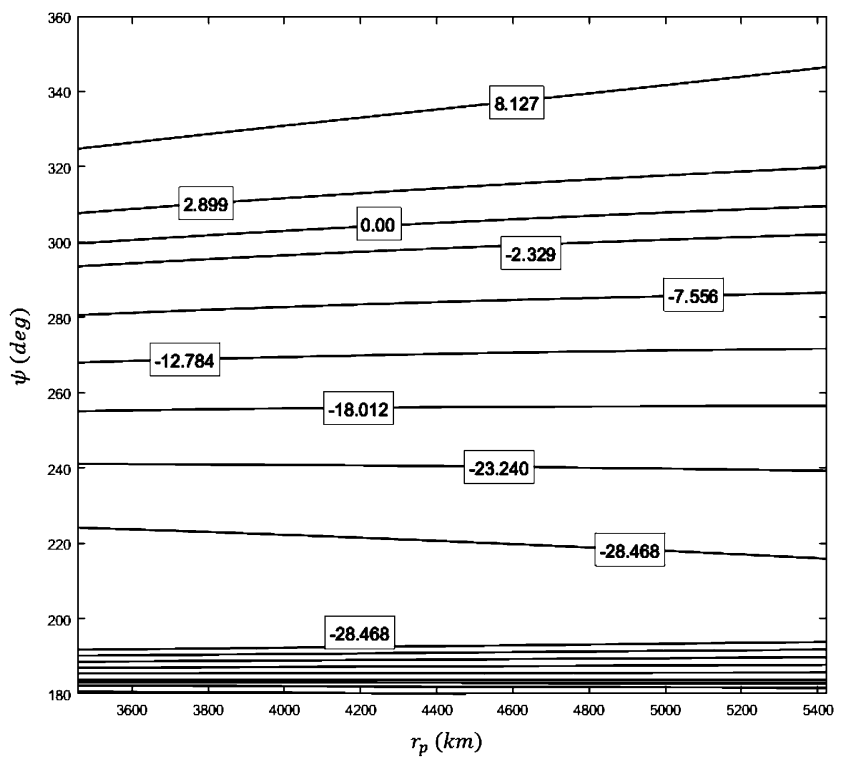

(b) Efficiency using $V_{\text {inf- }}=3.4 \mathrm{~km} / \mathrm{s}$

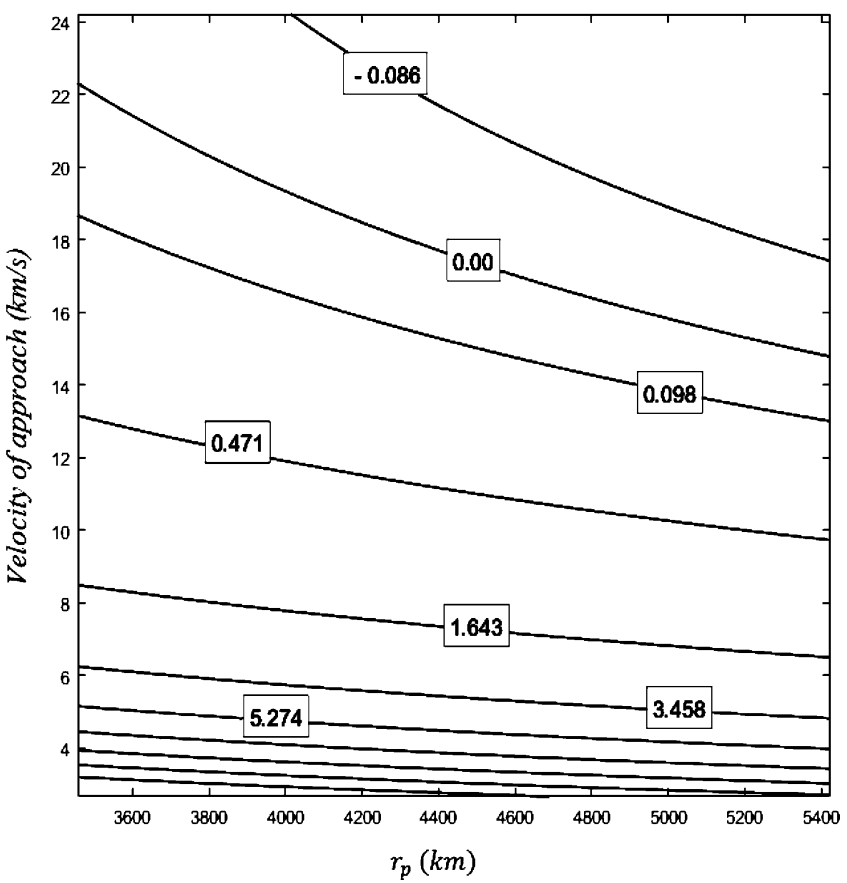

(d) Efficiency using $\psi=340^{\circ}$

Fig. 19 Analysis of the energy variation and efficiency in the energy gains region of the powered Swing-By for Sun-Mars system with $v=0^{\circ}$ and $\delta V=0.5 \mathrm{~km} / \mathrm{s}$

change due to this parameter when compared to the circular case.

The efficiency of the powered Swing-By, i.e., the amount of extra energy obtained by the spacecraft when the impulse is applied in the orbital periapsis of the spacecraft, compared to the pure gravity Swing-By maneuver followed by the application of the impulse outside the sphere of influence of the secondary body, was presented. The analysis was performed according to the gravitation parameter of secondary body $(\mu)$, periapsis radius $\left(r_{p}\right)$, orientation of the SwingBy maneuver $(\psi)$ and approach velocity $\left(V_{\text {inf- }}\right)$. The results mapped the regions where the powered Swing-By is efficient for a generic system.

The results were then applied to the Sun-Mercury and Sun-Mars examples, identifying the regions where the proposed maneuver is more efficient.

The results presented here can help mission designers to decide when this type of maneuver should be used or not. Of 


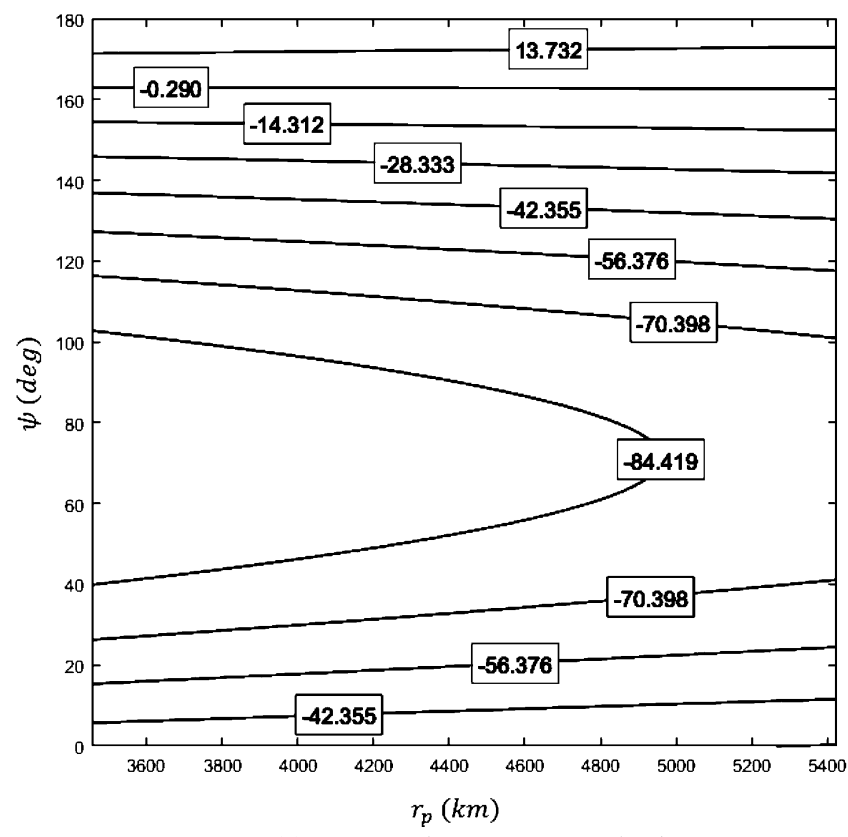

(a) $\Delta E_{\text {imp }}$ using $V_{\text {inf- }}=3.4 \mathrm{~km} / \mathrm{s}$

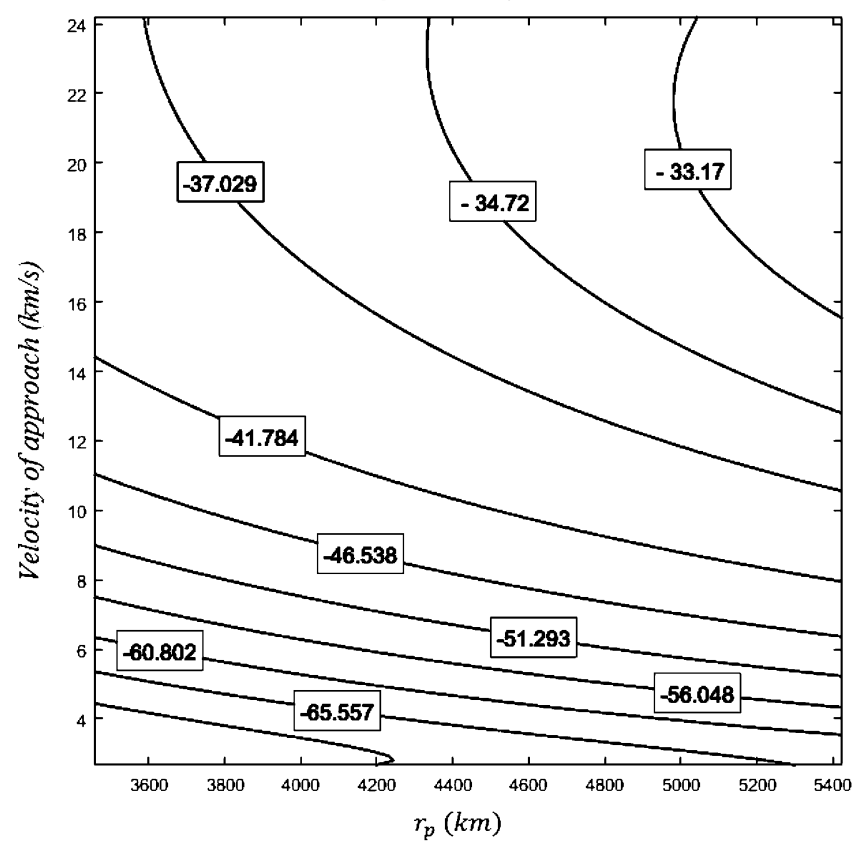

(c) $\Delta E_{\text {imp }}$ using $\psi=30^{\circ}$

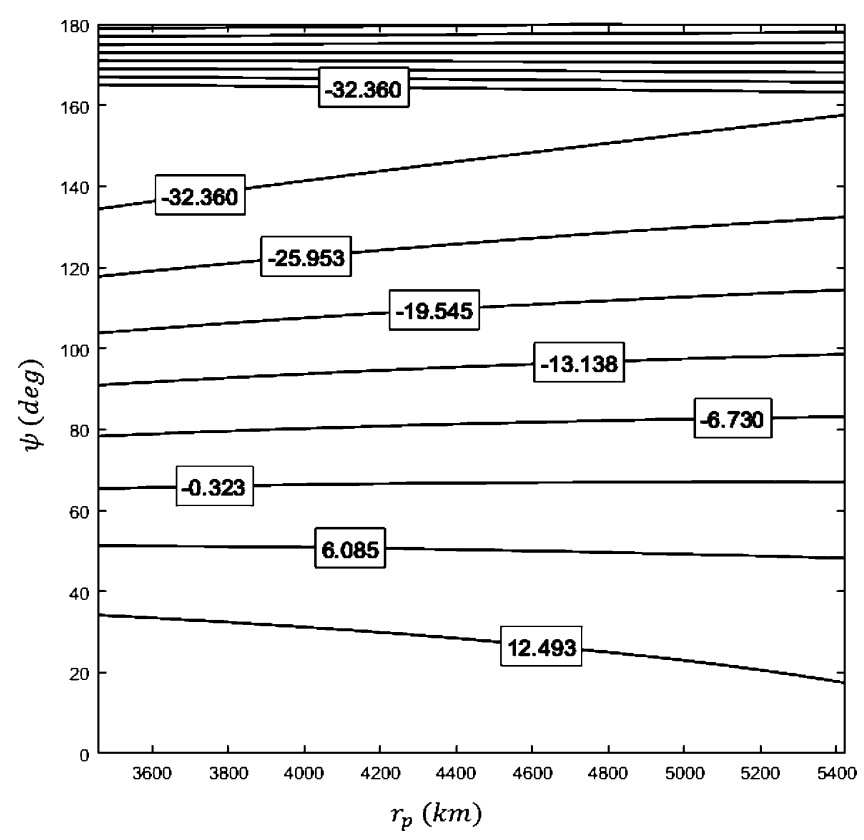

(b) Efficiency using $V_{\text {inf- }}=3.4 \mathrm{~km} / \mathrm{s}$

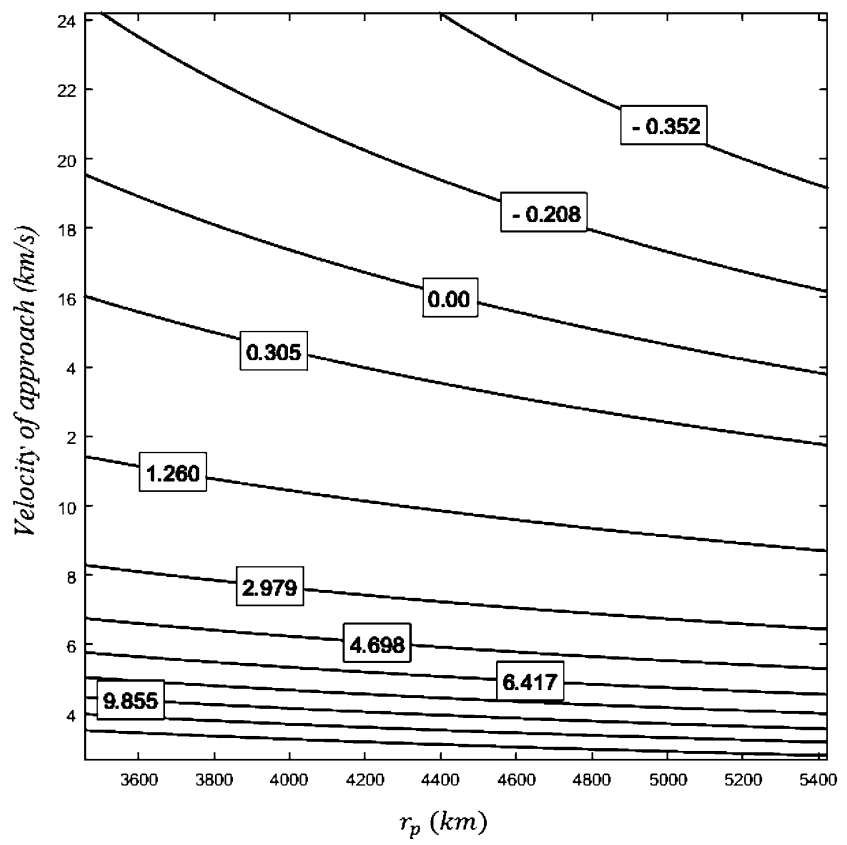

(d) Efficiency using $\psi=30^{\circ}$

Fig. 20 Analysis of the energy variation and efficiency in the energy losses region of the powered Swing-By for Sun-Mars system with $v=0^{\circ}$ and $\delta V=0.5 \mathrm{~km} / \mathrm{s}$

course Powered Swing-By maneuvers have risks involved, in particular due the possibility of errors in the thrust applied. The present paper do not recommend this type of maneuver in many situations, but concentrates in making a general study to identify when it is advantageous and how to perform the maneuvers. Therefore, the goal of the present paper was to increase the information available in the literature about the advantages of this type of maneuver. To use in real missions depends on the particularity of the mission.

Acknowledgements The authors wish to express their appreciation for the support provided by grants \# 406841/2016-0, 301338/2016-7 and 312813/2013-9 from the National Council for Scientific and Technological Development (CNPq); grants \#2016/14665-2, 2016/24561-0, 2016/23542-1, and 2017/04643-4 from São Paulo Research Foundation (FAPESP) and the financial support from the National Council for the Improvement of Higher Education (CAPES). 
Conflict of interest The authors declare that they have no conflict of interest.

\section{References}

Araujo, R.A.N., Winter, O.C., Prado, A.F.B.A., Vieira Martins, R.: Sphere of influence and gravitational capture radius: a dynamical approach. Mon. Not. R. Astron. Soc. 391(2), 675-684 (2008)

Barger, V., Olsson, M.: Classical Mechanics: A Modern Perspective. McGraw-Hill, New York (1973)

Bate, R.R., Mueller, D.D., White, J.E.: Fundamentals of Astrodynamics, pp. 333-334. Dover, New York (1971)

Broucke, R.A.: The celestial mechanics of gravity assist. In: AIAA/AAS Astrodynamics Conference, Minneapolis, MN, August (1988). https://doi.org/10.2514/6.1988-4220. AIAA paper $88-4220$

Byrnes, D.V., D'Amario, L.A.: A combined Halley flyby Galileo mission. In: AIAA/AAS Astrodynamics Conference, vol. 82 (1982). https://doi.org/10.2514/6.1982-1462

Casalino, L., Colasurdo, G., Pastrone, D.: Simple strategy for powered Swing-By. J. Guid. Control Dyn. 22(1), 156-159 (1999a)

Casalino, L., Colasurdo, G., Pasttrone, D.: Optimal low-thrust scape trajectories using gravity assist. J. Guid. Control Dyn. 22(5), 637$642(1999 b)$

D'Amario, L.A., Byrnes, D.V., Stanford, R.H.: A new method for optimizing multiple-flyby trajectories. J. Guid. Control Dyn. 4, 591 (1981). https://doi.org/10.2514/3.56115

D’Amario, L.A., Byrnes, D.V., Stanford, R.H.: Interplanetary trajectory optimization with application to Galileo. J. Guid. Control Dyn. 5, 465 (1982). https://doi.org/10.2514/3.56194

Dunne, J.A., Burgess, E.: The Voyage of Mariner 10. NASA SP 424 (1978)

Ferreira, A.F.S., Prado, A.F.B.A., Winter, O.C.: A numerical study of powered Swing-Bys around the Moon. Adv. Space Res. 56, 252272 (2015). https://doi.org/10.1016/j.asr.2015.04.016

Ferreira, A.F., Prado, A.F.B.A., Winter, O.C., Santos, D.P.S.: Effects of the eccentricity of the primaries in a powered Swing-By maneuver. Adv. Space Res. 59(8), 2071-2087 (2017a). https://doi.org/ 10.1016/j.asr.2017.01.033

Ferreira, A.F., Prado, A.F.B.A., Winter, O.C.: A numerical mapping of energy gains in a powered Swing-By maneuver. Nonlinear Dyn. 89(2), 791-818 (2017b). https://doi.org/10.1007/s11071017-3485-2

Ferreira, A.F., Prado, A.F.B.A., Winter, O.C., Santos, D.P.S.: Studying the energy variation in the powered Swing-By in the Sun-Mercury system. J. Phys. Conf. Ser. 911, 012007 (2017c)

Ferreira, A.F.S., Prado, A.F.B.A., Winter, O.C.: Analytical study of the Swing-By maneuver in an elliptical system. Astrophys. Space Sci. 363(2), 24 (2018)

Flandro, G.: Fast reconnaissance missions to the outer solar system utilizing energy derived from the gravitational field of Jupiter. Acta Astronaut. 12(4), 329 (1966)
Gomes, V.M., Prado, A.F.B.A.: Swing-By maneuvers for a cloud of particles with planets of the Solar System. WSEAS Trans. Appl. Theor. Mech. 3(11), 869-878 (2008)

Grard, R.: Mercury: the Messenger and BepiColombo missions a concerted approach to the exploration of the planet. Adv. Space Res. 38, 563 (2006). https://doi.org/10.1016/j.asr.2006.06.015

McConaghy, T.T., Debban, T.J., Petropoulos, A.E., Longuski, J.M.: Design and optimization of low-thrust gravity trajectories with gravity assist. J. Spacecr. Rockets 40, 380-387 (2003)

McNutt, R.L. Jr., Solomon, S.C., Grard, R., Novara, M., Mukai, T.: An international program for Mercury exploration: synergy of Messenger and Bepicolombo. Adv. Space Res. 33, 2126 (2004). https://doi.org/10.1016/S0273-1177(03)00439-3

McNutt, R.L. Jr., Solomon, S.C., Gold, R.E., Leary, J.C.: The messenger mission to Mercury: development history and early mission status. Adv. Space Res. 38, 564 (2006). https://doi.org/10.1016/ j.asr.2005.05.044

NASA. LCROSS - Lunar Crater Observation and Sensing SatelliteLCROSS Overview. Page Editor: Robert Garner (2009). Available in: https://www.nasa.gov/mission_pages/LCROSS/overview/ index.html

Negri, R.B., Prado, A.F.B.A., Sukhanov, A.: Studying the errors in the estimation of the variation of energy by the "patched-conics" model in the three-dimensional swing-by. Celest. Mech. Dyn. Astron. 1, 1 (2017)

Okutsu, M., Yam, C.H., Longuski, J.M.: Low-thrust trajectories to Jupiter via gravity assists from Venus, Earth and Mars. AIAA (2006), Paper 2006-6745

Pourtakdoust, S.H., Sayanjali, M.: Fourth body gravitation effect on the resonance orbit characteristics of the restricted three-body problem. Nonlinear Dyn. 76(2), 955-972 (2014)

Prado, A.F.B.A.: Powered Swing-By. J. Guid. Control Dyn. 19, 1142 1147 (1996). https://doi.org/10.2514/3.21756

Prado, A.F.B.A.: Close-approach trajectories in the elliptic restricted problem. J. Guid. Control Dyn. 20, 797-802 (1997). https:// doi.org/10.2514/2.4115

Prado, A.F.B.A.: A comparison of the "patched-conics" approach and the restricted problem for Swing-Bys. Adv. Space Res. 40, 113117 (2007). https://doi.org/10.1016/j.asr.2007.01.012

Qian, Y.J., Zhang, W., Yang, X.D., Yao, M.H.: Energy analysis and trajectory design for low-energy escaping orbit in Earth-Moon system. Nonlinear Dyn. 85(1), 463-478 (2016)

Sanchez, D.M., Howell, K.C., Prado, A.F.B.A.: On the dynamics of a spacecraft in the irregular Haumea-Hi'iaka binary system. Adv. Astronaut. Sci. 158, 3681-3697 (2016)

Santos, D.P.S., Prado, A.F.B.A., Casalino, L., Colasurdo, G.: Optimal trajectories towards near-Earth-objects using Solar electric propulsion (sep) and gravity assisted maneuver. J. Aerosp. Eng., Sci. Apl. 1(2), 51-64 (2008)

Zotos, E.E.: Classifying orbits in the restricted three-body problem. Nonlinear Dyn. 82(3), 1233-1250 (2015) 